TUM-EFT 75/15

\title{
FeynHelpers: Connecting FeynCalc to FIRE and Package-X
}

\author{
Vladyslav Shtabovenko, ${ }^{\mathrm{a}, *}$ \\ ${ }^{a}$ Technische Universität München, Physik-Department T30f, James-Franck-Str. 1, 85747 \\ Garching, Germany
}

\begin{abstract}
We present a new interface called FeynHelpers that connects FeynCalC, a MATHEMATICA package for symbolic semi-automatic evaluation of Feynman diagrams and calculations in quantum field theory (QFT) to PACKAGE$\mathrm{X}$ and FIRE. The former provides a library of analytic results for scalar 1-loop integrals with up to 4 legs, while the latter is a general-purpose tool for reduction of multi-loop scalar integrals using Integration-by-Parts (IBP) identities.
\end{abstract}

Keywords: High energy physics; Feynman diagrams; Loop integrals; Dimensional regularization; Renormalization; Effective field theories; Passarino-Veltman; IBP; FeynCalc; FIRE; Package-X;

\section{Introduction}

FEynCALC [1, 2] is firmly established in high energy physics (HEP) research and education as a flexible and easy to use tool for small and mediumsized symbolic QFT computations. Since the program by itself is mostly limited to algebraic manipulations, it is often used together with other packages, e.g. FeynArts, Looptools [3] or FeynRules [4]. While there is a built-in interface for converting amplitudes generated by FEYNARTS into valid FeynCalC input, for all the other tools one usually has to do such conversion by oneself.

As long as the other software is also written in Mathematica and the user has some programming experience with WoLfRAm language, it should

\footnotetext{
*E-mail address: v.shtabovenko@tum.de
} 
be not too difficult to create a simple converter that does the job for the current project. However, there is a big difference between writing limited code for personal use, with all its potential limitations and pitfalls and creating a robust, tested and well-maintained interface that can be useful for the HEP community.

The long-term maintenance is always important because subsequent versions of a particular tool may introduce changes in syntax, changes in normalization, new features or simply workflow improvements that require appropriate adjustments in the interface code. Also, the interface itself might require updates to be compatible with the latest releases of MATHEMATICA.

In this paper we would like to present the first stable ${ }^{1}$ public version of FeynHelpers, an interface that connects FeynCAlC to the MathematICA packages PACKAGE-X [6] and FIRE [7]. The combination of FEYNCALC and FEYNHELPERS allows one to obtain fully analytic results for most 1-loop amplitudes with up to 4 external legs and to rewrite many multi-loop amplitudes in terms of master loop integrals. Since the author of FEYNHELPERS is also the lead FEYNCALC developer, we believe that this interface can provide a stable long-term solution for using FEYNCALC, PACKAGE-X and FIRE in one framework, at least as long as the latter two packages are actively developed.

The main purpose for introducing FEYNHELPERS is not to compete with other established frameworks for loop calculations (e.g. FormCALC [3], GoSAm [8], FDC [9], GRACE [10], DiAna [11]) but rather to improve the usefulness of FEYNCALC for specific calculations, which require semi-automatic approach and therefore cannot be easily done using fully automatic all-in-one tools.

This paper is organized as follows. Section 2 briefly introduces MATHEMATICA packages PACKAGE-X and FIRE and explains their usefulness for automatized QFT calculations. Section 3 describes the technical implementation of the interface, which makes it possible to use both packages without interrupting the existing FEYNCALC session. The installation of the interface and its usage instructions are explained in Section 4. Section 5 presents four examples for calculations that can be significantly simplified when using FeynCalc and FeynHelpers. At the end we summarize the results and

\footnotetext{
${ }^{1}$ The development version including a link to the public code repository was first mentioned in 5.
} 
draw our conclusions in Section 6.

\section{Package-X and FIRE}

\subsection{Package- $X$}

Even though the Passarino-Veltman technique [12] for evaluation of 1loop tensor integrals was introduced almost four decades ago and alternative approaches are available (e.g. unitarity methods [13]), tensor decomposition is still widely used in many loop calculations. The key idea here is to convert all the occurring 1-loop tensor integrals into scalar ones, which are conventionally denoted as Passarino-Veltman coefficient functions. Thus, the calculation of an arbitrary 1-loop integral can be reduced to the evaluation of the resulting scalar functions. Often (e.g. in calculations of decay rates, cross sections or asymmetries) numerical evaluation of such functions is sufficient and many suitable tools for doing this are publicly available, e.g. FF [14], LoopTools [3], QCDLoop [15], OneLOop [16], Golem95C [17], PJFry [18] and Collier [19].

However, there are also cases (e.g. calculation of matching coefficients in effective field theories (EFTs), renormalization, etc.) where one would like to have fully analytic expressions for all the scalar functions that appear in the calculation. In general, it is desirable to have results for arbitrary kinematics, including zero Gram determinants but also for cases with vanishing and/or coinciding masses and scalar products of external momenta.

Although most of these results can be found in the literature (c.f. [20] and [21], as well as references in [6]), until recently there was no easy and convenient way to make use of them in automatic calculations. Public packages such as ANT [22] and LOOL [23] aimed to provide selected results for particular kinematical limits (e.g. vanishing external momenta or very large masses inside loops) are available since several years, but their applicability is limited to special cases.

This situation has changed with the release of PACKAGE-X [6], a MATHEMATICA package for semi-automatic 1-loop calculations. A unique feature of this package is the built-in library of analytic expressions for PassarinoVeltman functions with up to 4 legs and almost arbitrary kinematics. In a tedious work the author of PACKAGE-X has collected numerous analytic formulas from the literature and meticulously cross-checked everything by comparing with both analytic and numerical results. All this was then systematically implemented in an easy to use MATHEMATiCA package. Although 
PACKAGE-X can do much more than just analytically evaluate coefficient functions, in this work we would like to concentrate only on this aspect of the package.

Let us provide several examples for the usefulness of the built-in 1-loop library. When computing the 1-loop gluon self-energy in QCD (in Feynman gauge), the result depends on 3 coefficient functions:

$$
B_{0}\left(p^{2}, 0,0\right), \quad B_{0}\left(p^{2}, m^{2}, m^{2}\right), \quad A_{0}\left(m^{2}\right),
$$

where $p$ is the external momentum and $m$ is the mass of the quarks in the loops. Provided that PACKAGE-X has already been loaded via

$$
\begin{aligned}
\ln [1]:= & <<X^{c} \\
& \text { Package-X v2.0.1, by Hiren H. Patel } \\
& \text { For more information, see the guide }
\end{aligned}
$$

it is very easy to obtain explicit results for these coefficient functions

$$
\begin{aligned}
\ln [2]:= & l i=\{\operatorname{PVB}[0,0, p \cdot p, 0,0], \operatorname{PVB}[0,0, p \cdot p, m, m], \\
& \text { PVA }[0, m]\} \\
\text { Out }[2]= & \left\{B_{0}\left(p^{2} ; 0,0\right), B_{0}\left(p^{2} ; m, m\right), A_{0}(m)\right\} \\
\ln [3]:= & \text { res }=(\text { LoopRefine/@li); } \\
& \text { res } / / \text { TableForm }
\end{aligned}
$$

Out $[4] / /$ TableForm $=$

$$
\begin{aligned}
& \frac{1}{\tilde{\epsilon}}+\log \left(-\frac{\mu^{2}}{\mathrm{p}^{2}}\right)+2 \\
& \frac{1}{\tilde{\epsilon}}+\Lambda\left(\mathrm{p}^{2} ; \mathrm{m}, \mathrm{m}\right)+\log \left(\frac{\mu^{2}}{\mathrm{~m}^{2}}\right)+2 \\
& \mathrm{~m}^{2}\left(\frac{1}{\tilde{\epsilon}}+\log \left(\frac{\mu^{2}}{\mathrm{~m}^{2}}\right)\right)+\mathrm{m}^{2}
\end{aligned}
$$

where LoopRefine is the function that replaces coefficient functions with analytic expressions. Notice that the results returned by PACKAGE-X are computed using $D=4-2 \epsilon$. Furthermore, for brevity the overall factor $i / 16 \pi^{2}$ is omitted and $1 / \epsilon-\gamma_{E}+\log (4 \pi)$ is abbreviated by $1 / \tilde{\epsilon}$. For the 
same reason, the result for $B_{0}\left(p^{2}, m^{2}, m^{2}\right)$ is given as a function of $\Lambda$, which is defined as

$\Lambda\left(p^{2} ; m_{0}, m_{1}\right)=\frac{\sqrt{\lambda\left(p^{2}, m_{0}^{2}, m_{1}^{2}\right)}}{p^{2}} \ln \left(\frac{2 m_{0} m_{1}}{-p^{2}+m_{0}^{2}+m_{1}^{2}-\sqrt{\lambda\left(p^{2}, m_{0}^{2}, m_{1}^{2}\right)}}+i \varepsilon\right)$

with

$$
\lambda(a, b, c)=a^{2}+b^{2}+c^{2}-2 a b-2 a c-2 b c
$$

being the Källén function. The explicit expressions can be obtained by applying DiscExpand

$$
\begin{aligned}
& \ln [5]:=\operatorname{res}[[2]] / / \text { DiscExpand } \\
& \operatorname{Out}[5]=\frac{1}{\tilde{\epsilon}}+\frac{\sqrt{\mathrm{p}^{2}\left(\mathrm{p}^{2}-4 \mathrm{~m}^{2}\right)} \log \left(\frac{\sqrt{\mathrm{p}^{2}\left(\mathrm{p}^{2}-4 \mathrm{~m}^{2}\right)}+2 \mathrm{~m}^{2}-\mathrm{p}^{2}}{2 \mathrm{~m}^{2}}\right)}{\mathrm{p}^{2}}+\log \left(\frac{\mu^{2}}{\mathrm{~m}^{2}}\right)+2
\end{aligned}
$$

More examples and explanations can be found in the official tutoria $\left.\right|^{2}$.

A FEYNCALC user who is not interested in switching to a different package may naturally wonder about different possibilities to continue doing calculations in FEYNCALC and use PACKAGE-X only to obtain analytic results for the coefficient functions. One would, of course, like to automatize the whole procedure in such a way, that an easy and direct evaluation of PaVe functions with PACKAGE-X can be achieved without interrupting the current FEYNCALC session. This wish was indeed one of the original motivations for the development of FEYNHELPERS.

\subsection{FIRE}

Let us now turn to another important task that arises in many loop calculations, especially when one goes beyond 1-loop. It is well known that loop integrals with propagators that are raised to integer powers usually can be reduced to simpler ones by using integration-by-parts identities (IBP) [24]. The underlying formula that generates IBP relations is just a consequence of the divergence theorem in $D$-dimensional spaces

$$
\int \frac{d^{D} k_{1}}{(2 \pi)^{D}} \cdots \int \frac{d^{D} k_{n}}{(2 \pi)^{D}} \frac{\partial}{\partial k_{j}^{\mu}}\left(r^{\mu} \prod_{i} \frac{(p \cdot k)_{i}^{b_{i}}}{\left(q_{i}^{2}+m_{i}^{2}\right)^{a_{i}}}\right)=0, \quad a_{i}, b_{i} \in \mathbb{Z}_{0}
$$

\footnotetext{
${ }^{2}$ http://packagex.hepforge.org/tutorial-2.0.0.pdf
} 
where $q_{i}$ is a linear combination of loop and external momenta (with at least one loop momentum $),(p \cdot k)_{i}$ denote scalar products of a loop momentum with another loop or external momentum, $r^{\mu}$ can be a loop or an external momentum and $j$ can take any values between 1 and $n$. Finding a useful way to combine numerous IBP relations generated by Eq. (4) such, that many complicated integrals can be reduced to a small set of master integrals, is a nontrivial task that requires special algorithms like Laporta algorithm [25], Baikov's method [26] or S-bases [27] to name just a few of them. Often a combination of several algorithms is used to obtain the most efficient reduction for different families of loop integrals.

Several public packages (e.g. AIR [28], LiteRed [29], FIRE [7], REDuzE [30]) for doing IBP-reduction are available, but in this work we would like to focus specifically on FIRE. It is a specialist package that was developed for doing IBP-reduction in a very general and efficient way. FIRE comes with various options for fine-tuning the reduction procedure and extra utilities for using Fermat ${ }^{3}$ computer algebra system to speed up the calculations, and KyotoCabinet 4 to organize the integrals in a database. Furthermore, since version 5 of the package, one has a choice between doing reduction only by means of MATHEMATICA or via the (much faster) $\mathrm{C}++$ back-end. In the following we would like to provide several simple examples for using FIRE 5.2 with 1- and 2-loop integrals (c.f. [31] for the official manual).

Let us begin with the almost trivial case of $\int \frac{d^{D} q}{\left(q^{2}-m^{2}\right)^{\alpha}}$. The computation proceeds in 2 steps, where we first need to prepare start files that encode the information about the given topology

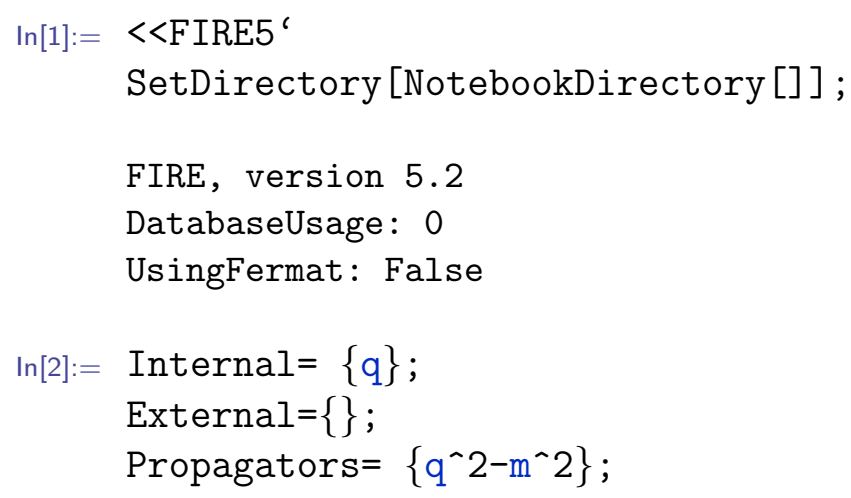




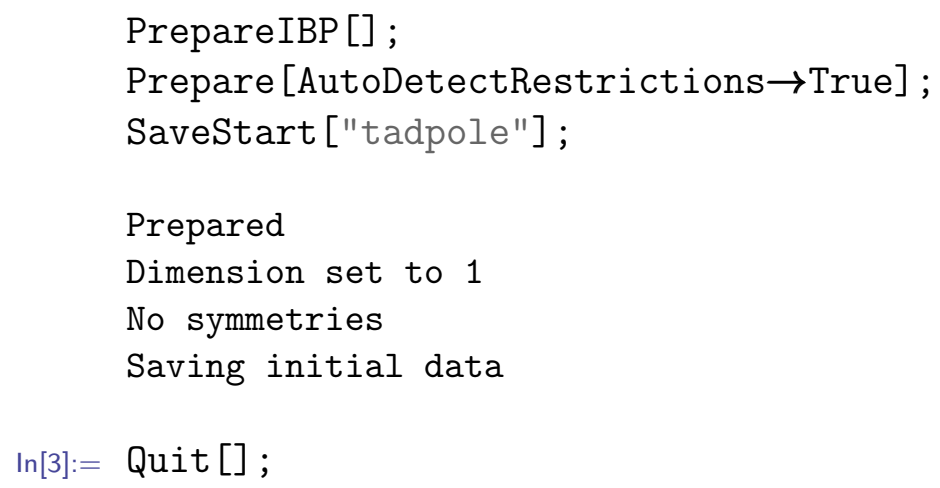

Notice that according to the official manual, after doing so we need to restart MATHEMATICA kernel, which is why the command Quit [] was added to the end of the code snippet. The information about the resulting integral family was saved to the file tadpole.start. In the next step we can perform the reduction by starting FIRE again, loading tadpole.start and supplying explicit integer numbers for $\alpha$

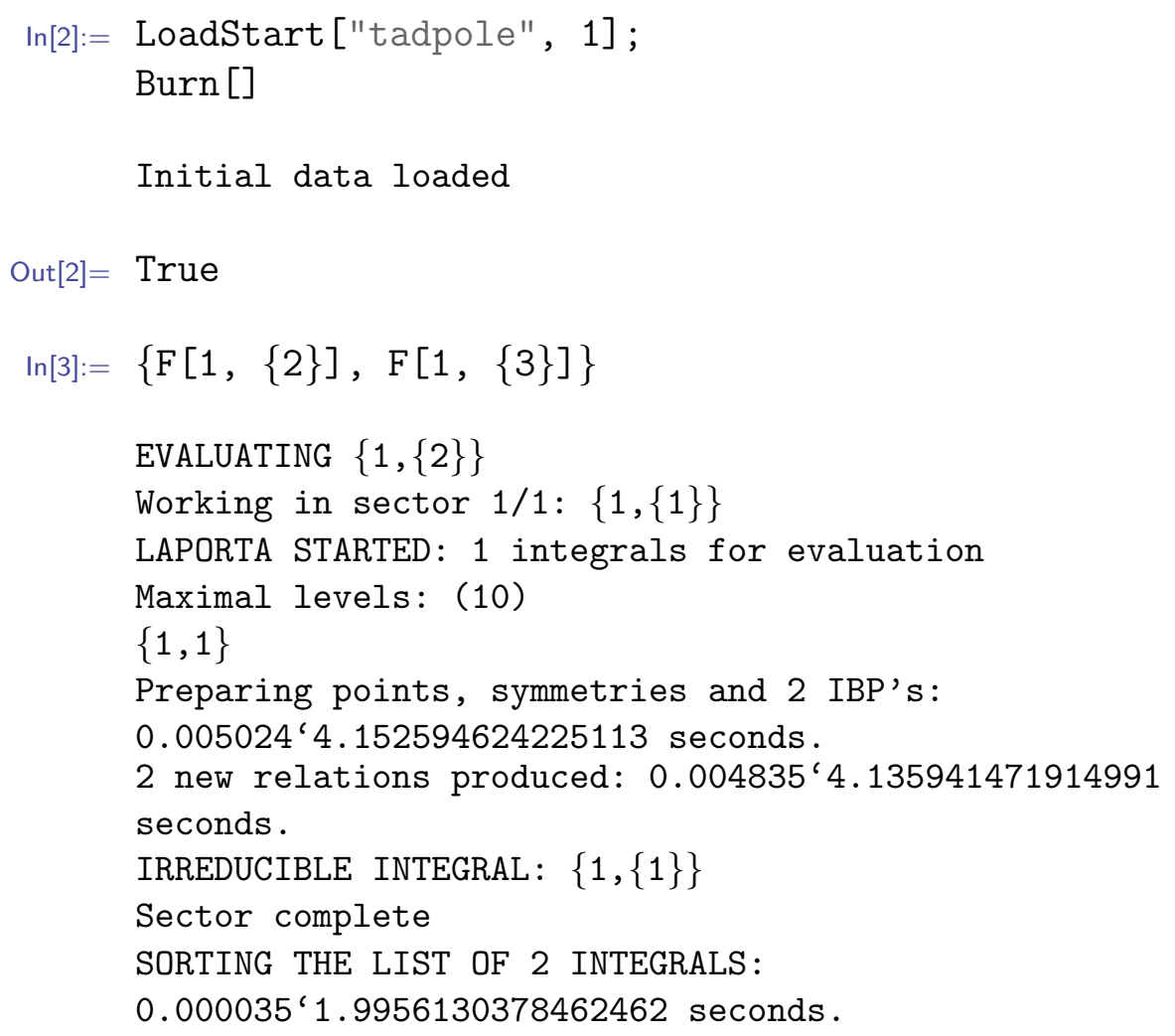




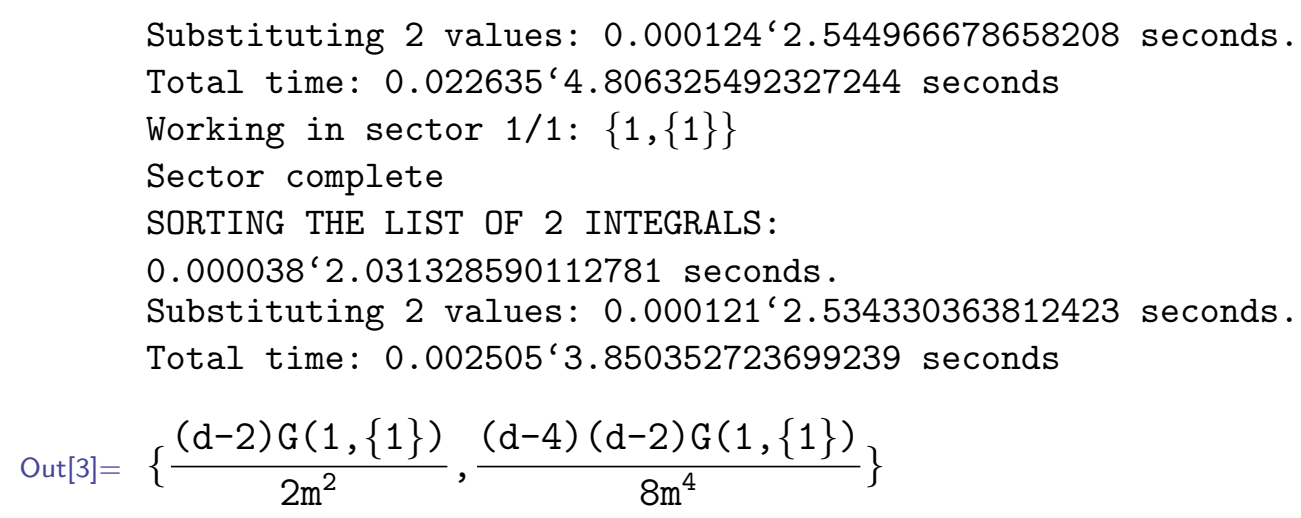

Here the first argument of $G$ identifies the integral family (the same integer was also used as the second argument of LoadStart), while the numbers in the brackets denote inverse powers of the original propagators. Hence, $G(1,\{1\})$ stands for $\int \frac{d^{D} q}{q^{2}-m^{2}}$.

Next, let us consider the integral $\int \frac{d^{D} q_{1} d^{D} q_{2}\left(p \cdot q_{2}\right)^{2}}{q_{1}^{2}\left[q_{2}^{2}\right]^{2}\left[\left(q_{1}-p\right)^{2}\right]^{3}\left[\left(q_{1}-q_{2}-p\right)^{2}\right]^{3}}$ which appears e.g. in the computation of the ghost self-energy at 2-loops in pure QCD. With the appropriate input for Internal, External and Propagators

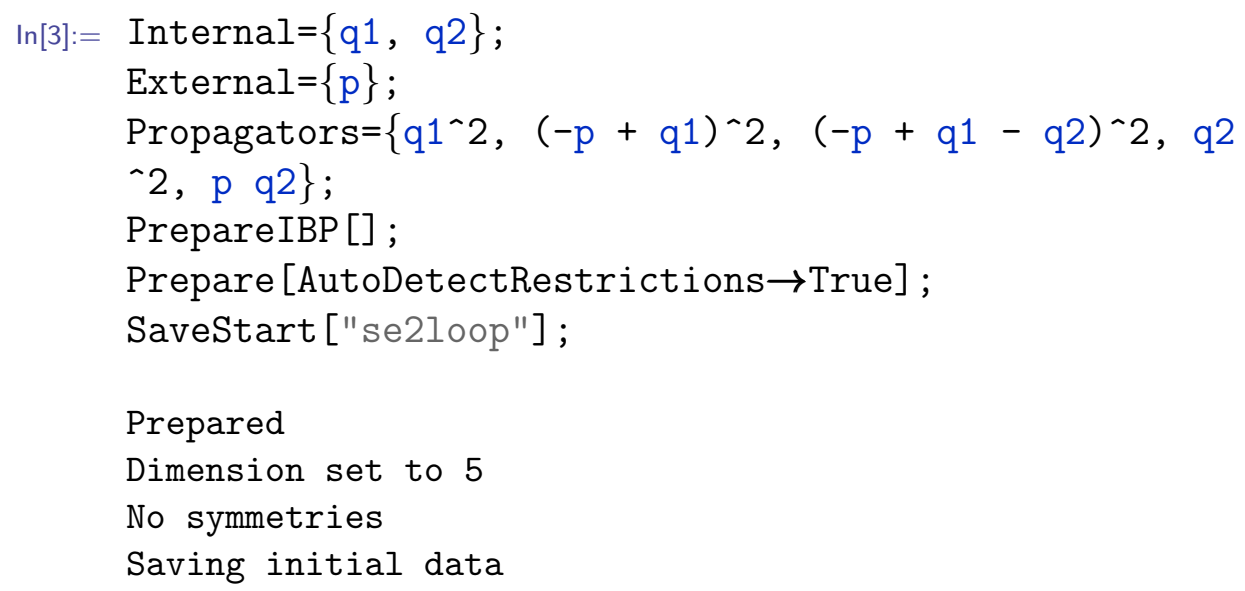

the result of the reduction reads

$$
\text { Out }[5]=-\frac{(3 d-10)(3 d-8)\left(d^{2}-12 d+38\right) G(1,\{1,0,1,1,0\})}{(d-10)(d-8)(d-6) p^{4}}
$$

In the above examples the reduction was carried out within MATHEMATICA in a reasonably small amount of time. For large calculations involving 
thousands or even millions of complicated multi-loop integrals, the performance of MATHEMATICA is usually not sufficient such that the use of the $\mathrm{C}++$ engine becomes mandatory. However, in this work we do not want to go into details of using FIRE in large scale higher loop calculations. The reason for this is that people interested in such projects mostly use in-house FORM [32] codes and would hardly consider FEYNCALC to be appropriate for the task.

On the other hand, small and medium-sized loop calculations that are feasible with FEYNCALC often may involve integrals that can be further simplified by using IBP-relations. Since FEYNCALC can perform such reduction only for 2-loop propagator-type integrals (via the TARCER [33] sub-package), the idea to utilize FIRE as a general IBP-reduction back-end for FEYNCALC appears tempting.

\section{Implementation}

Our goal is to be able to use PACKAGE-X and FIRE from an existing FEYNCALC session without worrying about different syntax, conventions, and normalizations. The interface should be seamless and easy to use, while still providing access to advanced configuration options. It is not intended to provide a pass-through for every possible function present in PACKAGE-X and FIRE, but rather to concentrate on those functions that appear to be most useful for FEynCALC users. For example, in case of PACKAGE-X we completely ignore the Lorentz and Dirac algebra modules of the package, as similar functionality is already present in FEYNCALC.

First observation to make is that we cannot naively load PACKAGE-X, FIRE and FEynCALC in the same Mathematica kernel. As the symbols DiracMatrix and Contract are present both in FEYNCALC and PACKAGE$\mathrm{X}$, one quickly runs into shadowing issues. It is also not possible to patch PACKAGE-X and change the conflicting names (as done with FEYNARTS), because the package is closed-source. Fortunately, it is possible to load just the 1-loop library of PACKAGE-X which does not contain any conflicting symbol names and was tested to safely coexist with FEYNCALC on the same Mathematica kerne $5^{5}$. On the other hand, the internal design of FIRE

\footnotetext{
${ }^{5}$ The author is grateful to Hiren Patel, the developer of PACKAGE-X, for explaining how to load only the OneLoop.m part of the package and for accounting for the compatibility with FeynHelpers while developing PACKAGe-X 2.0.
} 
which puts many of the symbols introduced by the package into the Global ' context of MATHEMATICA, makes it rather impractical to use it together with FEYNCALC within one kernel session. Even if that would be feasible, the necessity to quit the kernel before starting an IBP-reduction (c.f. Sec. 2.2 renders the whole idea of a seamless interface meaningless. Here one can take advantage of MATHEMATICA's parallel architecture that permits us to evaluate different parts of the calculation on separate kernels. This way it is possible to execute FIRE on a parallel kernel and safely communicate with it using the kernel that runs FeynCALC and FeynHelpers. Before a parallel kernel is started, for each loop integral the interface creates three files: FIREp1-intXXX.m, FIREp2-intXXX.m and FIRERepList-intXXX.m, where $\mathrm{XXX}$ denotes an integer number assigned to that integral. The first two files are required to run FIRE according to the instructions given in Sec. 2.2. They can be also evaluated outside of FEYNHELPERS, i.e. directly with MAthematica and FIRE. The third file contains replacement rules that are used to convert the result back into FEYNCALC notation. The files are located in FeynCalc/Database and can be examined for logging or debugging purposes. While this approach may appear too complicated and does induce time penalties for starting and stopping parallel kernels, it allows us to avoid unnecessary restarts of the main kernel, effectively prevents variable shadowing and does not require any modifications in the source code of FIRE.

Another subtlety to be taken into account when calling PACKAGE-X from FEYNCALC is the different normalization of 1-loop integrals. In Table 1, we summarize the existing ways to enter a 1-loop integral in FEYNCALC and PACKAGE-X.

\begin{tabular}{|l|l|}
\hline Command in FEYNCALC & Meaning \\
\hline FAD $[\{\mathrm{q}, \mathrm{m} 1\},\{\mathrm{q}-\mathrm{p}, \mathrm{m} 2\}]$ & $\int d^{D} q \frac{1}{\left[q^{2}-m 1^{2}\right]\left[(q-p)^{2}-m 2^{2}\right]}$ \\
$\operatorname{PaVe}\left[0,\{\mathrm{SPD}[\mathrm{p}, \mathrm{p}]\},\left\{\mathrm{m} 1^{2}, \mathrm{~m} 2^{2}\right\}\right]$ & $\int \frac{d^{D} q}{i \pi^{2}} \frac{1}{\left[q^{2}-m 1^{2}\right]\left[(q-p)^{2}-m 2^{2}\right]}$ \\
$\mathrm{BO}\left[\mathrm{SPD}[\mathrm{p}, \mathrm{p}], \mathrm{m} 1^{2}, \mathrm{~m} 2^{2}\right] 6$ & $\int \frac{d^{D} q}{i \pi^{2}} \frac{1}{\left[q^{2}-m 1^{2}\right]\left[(q-p)^{2}-m 2^{2}\right]}$ \\
\hline
\end{tabular}

\footnotetext{
${ }^{6} \mathrm{PaVe}$ is the standard way to enter coefficient functions in FEYNCALC. For historical reasons it is also possible to enter several functions directly, namely via A0, A00, B0, B1, B00, B11, C0 and D0.
} 


\begin{tabular}{|l|l|}
\hline Command in PACKAGE-X & Meaning \\
\hline LoopIntegrate $[1, \mathrm{q},\{\mathrm{q}, \mathrm{m} 1\},\{\mathrm{q}-\mathrm{p}, \mathrm{m} 2\}]$ & $\frac{(4 \pi)^{D / 2}}{i e^{-\gamma} E^{\epsilon}} \int \frac{d^{D} q}{(2 \pi)^{D}} \frac{1}{\left[q^{2}-m 1^{2}\right]\left[(q-p)^{2}-m 2^{2}\right]}$ \\
PVB $[0,0, \mathrm{p} \cdot \mathrm{p}, \mathrm{m} 1, \mathrm{~m} 2]$ & $\frac{(4 \pi)^{D / 2}}{i e^{-\gamma} E^{\epsilon}} \int \frac{d^{D} q}{(2 \pi)^{D}} \frac{1}{\left[q^{2}-m 1^{2}\right]\left[(q-p)^{2}-m 2^{2}\right]}$ \\
\hline
\end{tabular}

Table 1: Different ways of entering 1-loop integrals in FeynCALC and PACKaGe-X.

PACKAGE-X multiplies all loop integrals by $\left(\frac{i e^{-\gamma_{E}}}{(4 \pi)^{D / 2}}\right)^{-1}$ to conveniently remove the overall $i /\left(16 \pi^{2}\right)$ prefactor as well as terms with $\gamma_{E}$ and $\log (4 \pi)$ that accompany poles in $\epsilon$. To convert a coefficient function from FEYNCALC to PACKAGE-X and obtain the full result, we therefore need to multiply every PaVe-object by

$$
\left(\frac{i}{16 \pi^{2}}\right)^{-1} \frac{i \pi^{2}}{(2 \pi)^{D}} \stackrel{D=4-2 \epsilon}{=}(2 \pi)^{-2 \epsilon}
$$

and perform the substitutions

$$
\begin{aligned}
\frac{1}{\epsilon} & \rightarrow \frac{1}{\epsilon}-\gamma_{E}+\log (4 \pi) \\
\frac{1}{\epsilon^{2}} & \rightarrow \frac{1}{\epsilon^{2}}+\frac{1}{\epsilon}\left(-\gamma_{E}+\log (4 \pi)\right)+\frac{\gamma_{E}^{2}}{2}-\gamma_{E} \log (4 \pi)+\frac{1}{2} \log ^{2}(4 \pi)
\end{aligned}
$$

in the final result. All these steps are automatically handled by FEYNHELPERS, so that the user does not have to worry about different conventions. Let us also remark that in practical calculations with FEYNCALC it is often convenient to omit the $1 /(2 \pi)^{D}$ prefactor in front of 1-loop integrals. The prefactor is of course understood but for brevity not written down explicitly. FeYNHELPERS allows us to reintroduce the prefactor when evaluating loop integrals by using the option PaXImplicitPrefactor (c.f. Sec. 5).

When interfacing with FIRE we need to keep in mind that as of version 5.2 , the package is not capable to perform reduction of integrals with linearly dependent propagators or propagators that do not form a basis. This should not be regarded as a flaw of FIRE, since both decomposition into integrals with linearly independent propagators and completion of the propagator basis are operations that can be done in many ways. The choice often depends on the details of the calculation and especially on the preferred basis of master integrals. 
In Sec. 3.3 of [2] it was shown how one can handle these issues in FEYNCALC by using the functions ApartFF, FCLoopBasisFindCompletion, FCLoopBasis IncompleteQ and FCLoopBasisOverdeterminedQ. The interface takes advantage of this new functionality by always checking the completeness of the propagator basis before passing the integrals to FIRE. Automatic completion is performed when necessary, although it is also possible to supply a list of extra propagators by hand. For integrals with linearly dependent propagators the user receives a message to perform partial fractioning with ApartFF first.

Finally, let us briefly mention the existing alternatives to FEYNHELPERS. As far as PACKAGE-X is concerned, we are not aware of any other publicly available interface between this package and FEYNCALC. For FIRE several attempts to facilitate the transition from FEYNCALC exist. The APART ${ }^{7}$ [34, 35] package can do both partial fractioning (ApartAll) and basis completion (ApartComplete). It is also possible to convert loop integrals from FEYNCALC into the FIRE notation via FireArguments. Another package, called FARE $]^{8}[36$, is intended for tensor reduction of loop integrals. The resulting scalar integrals can be converted to the FIRE notation via FIREType. It is worth noting, however, that neither APART nor FARE currently make it possible to automatize the whole workflow, which includes not only converting the integrals but also preparing input files, running FIRE, fetching the results and sending them back to FEYNCALC, as it is done in FEYNHELPERS.

\section{Installation and Usage}

\subsection{Installation}

FeynHelpers is implemented as an add-on for FeynCalC 9.2 and above and is licensed under the General Public License (GPL) version 3. We would like to stress that this license applies only to the interface itself, but not to PACKAGE-X and FIRE. Their licensing conditions are outlined on the corresponding websites? Questions with regard to the usage of FeynHelpers can be posted to the FeynCALC mailing list ${ }^{10}$, To install

\footnotetext{
$7^{7}$ https://github.com/F-Feng/APart

8 https://sourceforge.net/projects/feyntoolfare

${ }^{9}$ http://packagex.hepforge.org

http://science.sander.su/FIRE.htm

10 https://feyncalc.github.io/forum
} 
FeynHelpers using the online installer, it is sufficient to evaluate

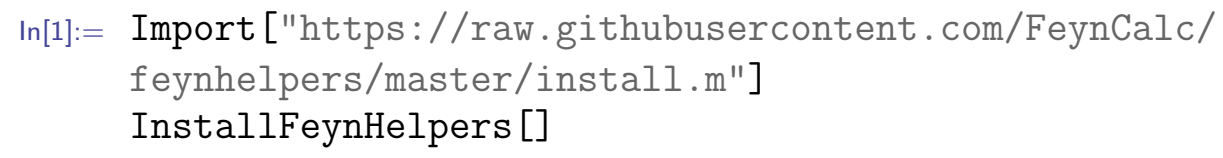

The installer automatically suggests downloading and installing PACKAGE$\mathrm{X}$ and FIRE if the packages are not found. To use the add-on, one should specify that it should be loaded before starting FEYNCALC.

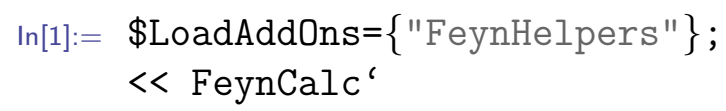

Essentially there are only two functions that handle all the communication between FEYNCALC and the two other packages: PaXEvaluate and FIREBurn.

\subsection{PaXEvaluate}

PaXEvaluate is the main function of the PACKAGE-X-interface. It works on scalar loop integrals without any loop-momentum dependent scalar products in the numerator and on Passarino-Veltman functions. If one wants to obtain only the UV- or the IR-divergent part of the result, one can use PaXEvaluateUV and PaXEvaluateIR. Finally, PaXEvaluateUVIRSplit returns the full result with an explicit distinction between $\epsilon_{\mathrm{UV}}$ and $\epsilon_{\mathrm{IR}}$. All four functions share the same set of options.

PaXEvaluate $[\operatorname{expr}, q]$ converts all the scalar 1-loop integrals with loop momentum $q$ in expr to Passarino-Veltman functions, which are then analytically evaluated using PACKAGE-X. Both UV- and IRsingularities are regulated with $\epsilon$. If $q$ is omitted, no conversion is done and only already present Passarino-Veltman functions are evaluated. 


$\begin{array}{|ll|}\text { PaXEvaluateUV }[\text { expr, }] & \text { like PaXEvaluate, but only the } \\ & 1 / \epsilon_{\mathrm{UV}} \text {-piece of the result is } \\ & \text { returned. } \\ \text { PaXEvaluateIR }[\text { expr, }]] & \text { like PaXEvaluate, but only } \\ & \text { the } 1 / \epsilon_{\mathrm{IR}} \text {-piece of the result is } \\ & \text { returned. } \\ & \\ \text { PaXEvaluateUVIRSplit }[\text { expr, } q] & \text { like PaXEvaluate, but with } \\ & \text { the explicit distinction between } \\ & 1 / \epsilon_{\mathrm{UV}} \text { and } 1 / \epsilon_{\mathrm{IR}} \text { in the final } \\ & \text { result. }\end{array}$

\begin{tabular}{|lll|}
\hline Option & Default value & Description \\
\hline Trlect & True & $\begin{array}{l}\text { whether the result should be } \\
\text { collected with respect to scalar } \\
\text { products metric tensors and } \\
\text { the Levi-Civita tensors. }\end{array}$ \\
Dimension & D & $\begin{array}{l}\text { the symbol that denotes } D- \\
\text { dimensions in the loop inte- } \\
\text { grals. }\end{array}$ \\
FCE & False & $\begin{array}{l}\text { whether } \\
\text { should be converted into } \\
\text { FeynCalcExternal-notation. }\end{array}$ \\
FCVerbose & False & $\begin{array}{l}\text { allows us to activate the de- } \\
\text { bugging output. }\end{array}$ \\
FinalSubstitutions & \{\} & $\begin{array}{l}\text { list of replacements to be ap- } \\
\text { plied to the final result. }\end{array}$
\end{tabular}




\begin{tabular}{|c|c|c|}
\hline PaVeAutoOrder & True & $\begin{array}{l}\text { automatic ordering of argu- } \\
\text { ments inside FeynCalc's PaVe } \\
\text { functions. }\end{array}$ \\
\hline PaVeAutoReduce & True & $\begin{array}{l}\text { automatic reduction of certain } \\
\mathrm{PaVe} \text { functions into simpler } \\
\text { ones. }\end{array}$ \\
\hline PaXCOExpand & False & $\begin{array}{l}\text { whether the full analytic result } \\
\text { for the } C_{0} \text { function should be } \\
\text { inserted. }\end{array}$ \\
\hline PaXD0Expand & False & $\begin{array}{l}\text { whether the full analytic result } \\
\text { for the } D_{0} \text { function should be } \\
\text { inserted. }\end{array}$ \\
\hline PaXDiscExpand & True & $\begin{array}{l}\text { whether the DiscB function } \\
\text { of PACKAGE-X should be re- } \\
\text { placed with its explicit expres- } \\
\text { sion. }\end{array}$ \\
\hline PaXExpandInEpsilon & True & $\begin{array}{l}\text { whether the final re- } \\
\text { sults multiplied by } \\
\text { PaXImplicitPrefactor } \\
\text { should be expanded around } \\
4-2 \epsilon \text {. }\end{array}$ \\
\hline PaXImplicitPrefactor & 1 & $\begin{array}{l}\text { a } D \text {-dependent prefactor that } \\
\text { multiplies the final result. }\end{array}$ \\
\hline PaXKallenExpand & True & $\begin{array}{l}\text { whether the Kallen } \lambda \text { function } \\
\text { of PACKAGE-X should be re- } \\
\text { placed with its explicit expres- } \\
\text { sion. }\end{array}$ \\
\hline
\end{tabular}




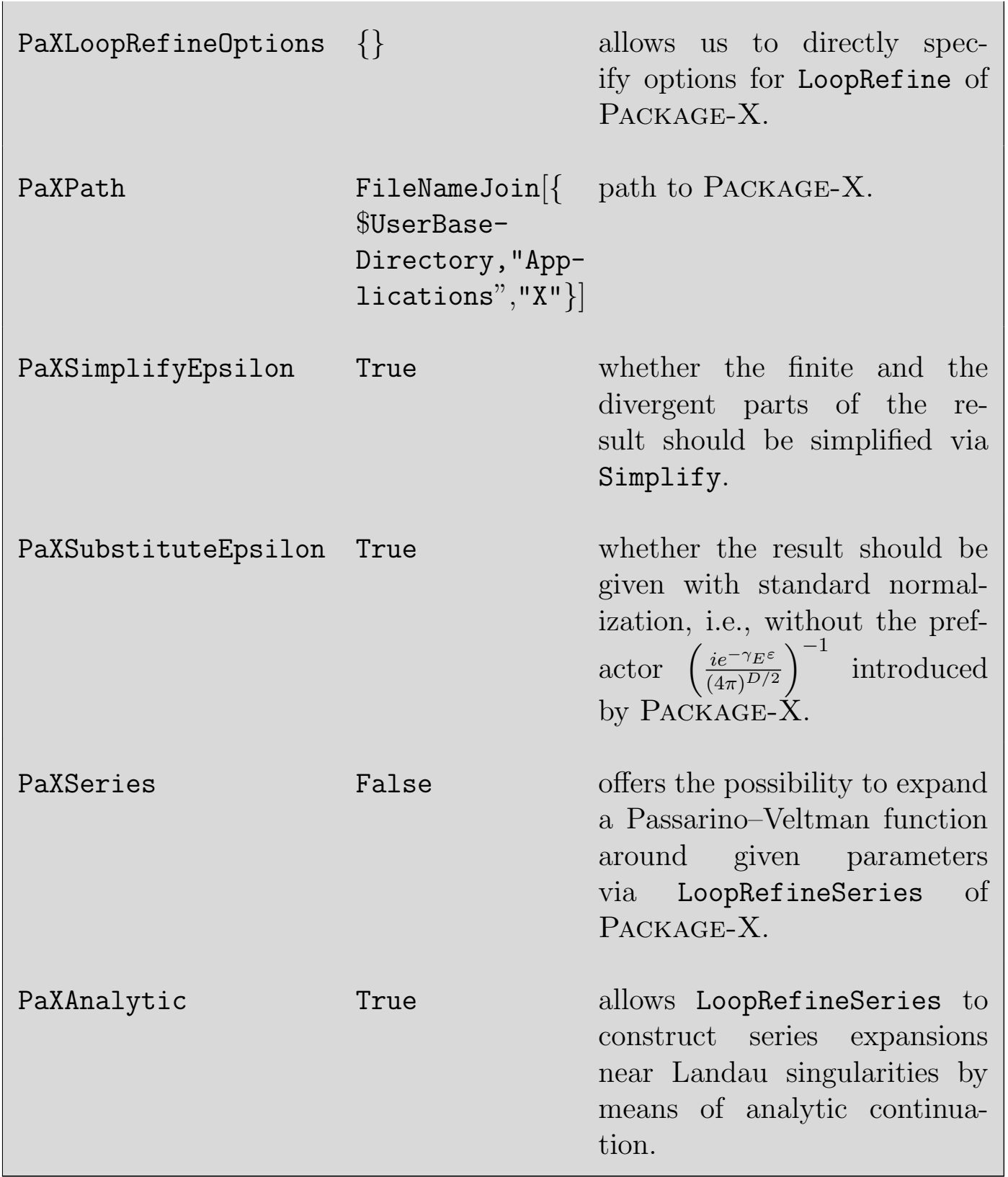

PaXEvaluate is designed in such a way that the function requires only a minimal amount of user input. For example, to compute the integral $\int \frac{d^{D} q}{(2 \pi)^{D}} \frac{1}{q^{2}-m^{2}}$ it is sufficient to write 


$$
\begin{aligned}
\ln [2]:= & \text { int=PaXEvaluate }[\mathrm{FAD}[\{\mathrm{q}, \mathrm{m}\}], \mathrm{q}, \mathrm{PaXImplicitPrefactor} \\
& \left.\rightarrow 1 /(2 \mathrm{Pi})^{\wedge} \mathrm{D}\right] \\
\text { Out }[2]= & \frac{\mathrm{im}^{2}}{16 \pi^{2} \varepsilon}-\frac{i \mathrm{~m}^{2}\left(-\log \left(\frac{\mu^{2}}{\mathrm{~m}^{2}}\right)+\gamma-1-\log (4 \pi)\right)}{16 \pi^{2}}
\end{aligned}
$$

where the first argument is our integral, the second is the loop momentum and the third is the option to specify the normalization. To make the result look more compact, we can introduce the abbreviation $\Delta \equiv 1 / \epsilon-\gamma_{E}+\log (4 \pi)$. This can be done with the FEYNCALC function FCHideEpsilon

$$
\begin{gathered}
\operatorname{In}[3]:=\text { int//FCHideEpsilon } \\
\text { Out[3] }=\frac{i \Delta \mathrm{m}^{2}}{16 \pi^{2}}+\frac{i \mathrm{~m}^{2}\left(\log \left(\frac{\mu^{2}}{\mathrm{~m}^{2}}\right)+1\right)}{16 \pi^{2}}
\end{gathered}
$$

In practical calculations one is usually interested in the evaluation of different Passarino-Veltman functions. In this case it is not needed to specify the loop momentum, such that to compute e.g. $B_{0}\left(p^{2}, 0, m^{2}\right)$ we use

$$
\begin{aligned}
\operatorname{In}[4]:= & \text { PaXEvaluate }\left[\mathrm{BO}\left[\operatorname{SPD}[\mathrm{p}, \mathrm{p}], 0, \mathrm{~m}^{\wedge} 2\right]\right] \\
\text { Out }[4] & =\frac{1}{\varepsilon}+\log \left(\frac{\mu^{2}}{\pi \mathrm{m}^{2}}\right)-\frac{\mathrm{m}^{2} \log \left(\frac{\mathrm{m}^{2}}{\mathrm{~m}^{2}-\mathrm{p}^{2}}\right)}{\mathrm{p}^{2}}+\log \left(\frac{\mathrm{m}^{2}}{\mathrm{~m}^{2}-\mathrm{p}^{2}}\right)-\gamma+2
\end{aligned}
$$

PACKAGE-X can also expand coefficient functions in their parameters (masses or external momenta). To expand $B_{0}\left(p^{2}, 0, m^{2}\right)$ around $p^{2}=m^{2}$ up to first order with PaXEvaluate we first need to assign an arbitrary symbolic value to the scalar product $p^{2}$, e.g. pp

$$
\ln [5]:=\operatorname{SPD}[\mathrm{p}, \mathrm{p}]=\mathrm{pp} \text {; }
$$

Then we use the option PaXSeries to specify the expansion parameters and activate the option PaXAnalytic to ensure that the derivatives of loop integrals are taken appropriately

$$
\begin{aligned}
\operatorname{In}[6]:= & \text { PaXEvaluate }\left[\mathrm { BO } [ \operatorname { S P D } [ \mathrm { p } , \mathrm { p } ] , 0 , \mathrm { m } ^ { \wedge } 2 ] , \text { PaXSeries } \rightarrow \left\{\left\{\mathrm{pp}, \mathrm{m}^{\wedge} 2,1\right.\right.\right. \\
& \}\}, \text { PaXAnalytic } \rightarrow \text { True }] \\
\text { Out }[6]= & \frac{3 \mathrm{~m}^{2}-\mathrm{pp}}{2 \varepsilon \mathrm{m}^{2}}-\frac{\left(3 \mathrm{~m}^{2}-\mathrm{pp}\right)\left(-\log \left(\frac{\mu^{2}}{\mathrm{~m}^{2}}\right)+\gamma-2+\log (\pi)\right)}{2 \mathrm{~m}^{2}}
\end{aligned}
$$


If we are interested only in the UV-part of this series, it is sufficient to replace PaXEvaluate with PaXEvaluateUV

$$
\begin{aligned}
\ln [7]:= & \text { PaXEvaluateUV }\left[B 0 [ \operatorname { S P D } [ \mathrm { p } , \mathrm { p } ] , 0 , \mathrm { m } ^ { \wedge } 2 ] , \text { PaXSeries } \rightarrow \left\{\left\{\mathrm{pp}, \mathrm{m}^{\wedge} 2,1\right.\right.\right. \\
& \}\}, \text { PaXAnalytic } \rightarrow \text { True }] \\
\text { Out }[7]= & \frac{1}{\varepsilon_{\mathrm{UV}}}
\end{aligned}
$$

Similarly, we can also obtain the IR-part of the series

$$
\begin{aligned}
\ln [8]:= & \text { PaXEvaluateIR }\left[\text { BO } [ \operatorname { S P D } [ \mathrm { p } , \mathrm { p } ] , 0 , \mathrm { m } ^ { \wedge } 2 ] , \text { PaXSeries } \rightarrow \left\{\left\{\mathrm{pp}, \mathrm{m}^{\wedge} 2,1\right.\right.\right. \\
& \}\}, \text { PaXAnalytic } \rightarrow \text { True }] \\
\text { Out }[8]= & \frac{\mathrm{m}^{2}-\mathrm{pp}}{2 \mathrm{~m}^{2} \varepsilon_{\mathrm{IR}}}
\end{aligned}
$$

Finally, PaXEvaluateUVIRSplit returns the result with explicit distinction between UV and IR singularities

$$
\begin{aligned}
\operatorname{In}[9]:= & \text { PaXEvaluateUVIRSplit }\left[\mathrm{BO}\left[\mathrm{SPD}[\mathrm{p}, \mathrm{p}], 0, \mathrm{~m}^{\wedge} 2\right],\right. \text { PaXSeries } \\
& \left.\rightarrow\left\{\left\{\mathrm{pp}, \mathrm{m}^{\wedge} 2,1\right\}\right\}, \text { PaXAnalytic } \rightarrow \text { True }\right] \\
\text { Out }[9]= & \frac{\mathrm{m}^{2}-\mathrm{pp}}{2 \mathrm{~m}^{2} \varepsilon_{\mathrm{IR}}}-\frac{\left(3 \mathrm{~m}^{2}-\mathrm{pp}\right)\left(-\log \left(\frac{\mu^{2}}{\mathrm{~m}^{2}}\right)+\gamma-2+\log (\pi)\right)}{2 \mathrm{~m}^{2}}+\frac{1}{\varepsilon_{\mathrm{UV}}}
\end{aligned}
$$

A comment is in place here. It is well known that in dimensional regularization (DR) it is possible to regularize both ultraviolet (UV) and infrared (IR) divergences with the same regulator $\epsilon$. To disentangle both types of divergences one can use different regulators, i.e. keep $\epsilon$ for the UV divergences and regulate the IR divergences with a fictitious mass. On the other hand, it is also possible to distinguish between UV and IR divergences in DR by using $\epsilon_{\mathrm{UV}}$ and $\epsilon_{\mathrm{IR}}$, as is done e.g., in [37]. Such a distinction is often useful in matching calculations for EFTs, but also in renormalization calculations. With this prescription the rule that scaleless integrals vanish in DR does not hold anymore. For example, the logarithmically divergent scaleless 1-loop integral

$$
\int \frac{d^{D} l}{(2 \pi)^{D}} \frac{1}{l^{4}}=\int \frac{d^{D} l}{(2 \pi)^{D}}\left(\frac{1}{l^{2}\left(l^{2}-m^{2}\right)}-\frac{m^{2}}{l^{4}\left(l^{2}-m^{2}\right)}\right)
$$


cannot be set to zero, as it is proportional to $1 / \epsilon_{\mathrm{UV}}-1 / \epsilon_{\mathrm{IR}}$. FEYNCALC 9.2 features a new global option \$KeepLogDivergentScalelessIntegrals, which prevents the internal functions from setting such scaleless 1-loop integrals to zero. Together with PaXEvaluateUVIRSplit this can be used to consistently distinguish between UV and IR singularities regulated dimensionally at 1-loop. For example, we can see that after setting

$$
\ln [10]:=\text { \$KeepLogDivergentScalelessIntegrals=True; }
$$

the integral $\int \frac{d^{D} l}{(2 \pi)^{D}} \frac{1}{l^{4}}$ does not vanish anymore but evaluates to

$$
\begin{aligned}
& \operatorname{In}[11]:=\text { PaXEvaluateUVIRSplit }[F A D[\{1,0,2\}], 1 \text {, } \\
& \text { PaXImplicitPrefactor->1/(2Pi)^D] } \\
& \text { Out }[11]=\frac{i}{16 \pi^{2} \varepsilon_{\mathrm{UV}}}-\frac{i}{16 \pi^{2} \varepsilon_{\mathrm{IR}}}
\end{aligned}
$$

Notice that this prescription is implemented in FEYNCALC for 1-loop integrals only. Furthermore, to ensure that the results are consistent, one should not use FIREBurn, as by default FIRE always sets all the scaleless integrals to zero.

\subsection{FIREBurn}

FIREBurn is the main function of the FIRE-interface. It reduces scalar multi-loop integrals to simpler ones using IBP-techniques.

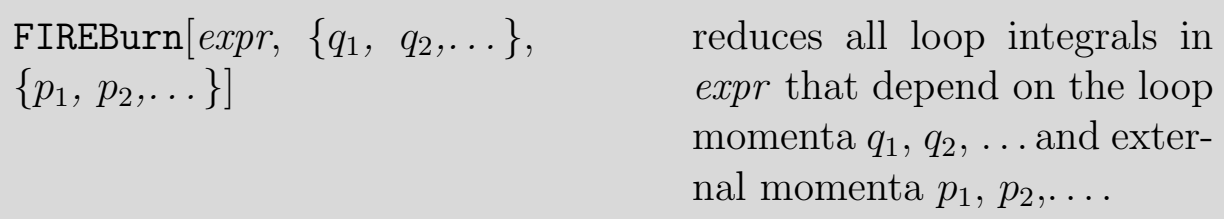

\section{Option Default value Description}




\begin{tabular}{|c|c|c|}
\hline Collect & True & $\begin{array}{l}\text { whether the result should be } \\
\text { collected with respect to the } \\
\text { loop integrals. }\end{array}$ \\
\hline FCE & False & $\begin{array}{l}\text { whether the result } \\
\text { should be converted into } \\
\text { FeynCalcExternal-notation. }\end{array}$ \\
\hline FCLoopIBPReducableQ & False & $\begin{array}{l}\text { whether FIRE should try to } \\
\text { reduce every loop integral in } \\
\text { the expression (default) or only } \\
\text { those that contain propagators } \\
\text { raised to integer powers. }\end{array}$ \\
\hline FCVerbose & False & $\begin{array}{l}\text { allows us to activate the de- } \\
\text { bugging output. }\end{array}$ \\
\hline FIREAddPropagators & Automatic & $\begin{array}{l}\text { whether extra propagators } \\
\text { with zero powers needed to } \\
\text { complete the basis should be } \\
\text { added automatically or by } \\
\text { hand. }\end{array}$ \\
\hline
\end{tabular}




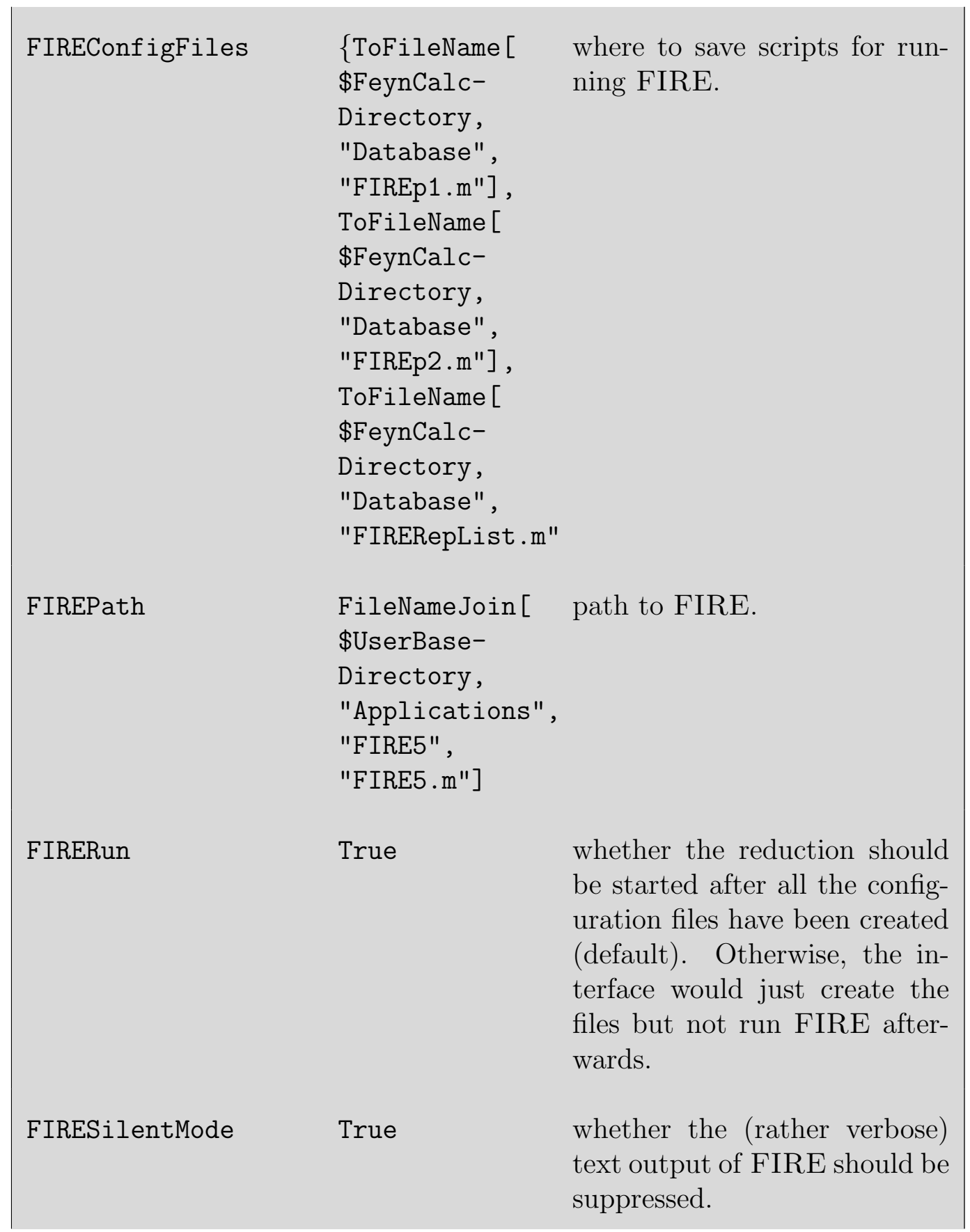


FIREStartFile

Timing
ToFileName [

\$FeynCalc-

Directory,

"Database",

"FIREStartFile"

True where to save start file for the FIRE engine.

informs the user about the progress of the IBP-reduction.

The function requires only three arguments, which are the input expression, the list of loop momenta and the list of external momenta. For example, to IBP-reduce the 1-loop integral $\int \frac{d^{D} l}{\left[l^{2}\right]^{2}\left[(l-p)^{2}-m^{2}\right]^{2}}$ we need to enter

$$
\begin{aligned}
\operatorname{In}[12]:= & \text { FIREBurn }[F A D[\{1,0,2\},\{1-p, m, 2\}],\{1\},\{p\}] \\
\operatorname{Out}[12]= & \frac{(D-2)\left(2 \mathrm{D} \mathrm{m}^{2}-9 \mathrm{~m}^{2}-\mathrm{pp}\right)}{2 \mathrm{~m}^{2}\left(\mathrm{~m}^{2}-\mathrm{pp}\right)^{3}\left((1-p)^{2}-\mathrm{m}^{2}\right)}-\frac{(\mathrm{D}-3)\left(D \mathrm{~m}^{2}+\mathrm{D} \mathrm{pp}-4 \mathrm{~m}^{2}-6 \mathrm{pp}\right)}{\left(\mathrm{m}^{2}-\mathrm{pp}\right)^{3} \mathrm{I}^{2} \cdot\left((1-p)^{2}-\mathrm{m}^{2}\right)}
\end{aligned}
$$

If the integral has no dependence on external momenta, as for example the 3-loop integral $\int \frac{d^{D} q_{1} d^{D} q_{2} d^{D} q_{3}}{\left[q 1^{2}-m^{2}\right]^{2}\left[\left(q_{1}+q_{3}\right)^{2}-m^{2}\right]\left[\left(q_{2}-q_{3}\right)^{2}\right]\left[q_{2}^{2}\right]^{2}}$, then the list of external momenta should be left empty

$$
\begin{aligned}
& \ln [13]:= \text { FIREBurn }[F A D[\{q 1, m, 2\},\{q 1+q 3, m\},\{q 2-q 3\},\{q 2,0,2\}],\{q 1, \\
&q 2, q 3\},\{\}] \\
& \text { Out }[13]=-\frac{(D-3)(3 D-10)(3 D-8)}{16(2 D-7) m^{4}\left(q 1^{2}-m^{2}\right) \cdot q 2^{2} \cdot(q 2-q 3)^{2} \cdot\left((q 1+q 3)^{2}-m^{2}\right)}
\end{aligned}
$$

In the current implementation of the interface to FIRE, each loop integral is evaluated separately, which is of course rather inefficient. Unfortunately, FEYNCALC is still not able to automatically recognize multi-loop integrals that belong to the same topology, which is undoubtedly a crucial requirement to make the program more useful in multi-loop calculations. Despite this limitation, we believe that FIREBurn can be well employed in smaller calculations with a low number of loops, where the IBP-reduction makes it possible to arrive to simpler results. 


\section{Examples}

So far we explained how to use FEynHelpers to evaluate single loop integrals. To demonstrate the usefulness of the interface in more realistic scenarios, we will provide four examples that make extensive use of the new functions introduced with FEYNHELPERS. The complete working codes for these calculations can be found in the accompanying MATHEMATICA notebooks QED-Renormalization.nb, NRQCD.nb, HiggsDecay.nb and QED-TwoLoop-SelfEnergies.nb. These codes are shipped together with FEYNHELPERs ${ }^{11}$ and can be also viewed online ${ }^{12}$, To avoid cluttering up this paper we will not copy the full code here but rather merely explain the most important steps and present the final results.

\subsection{Renormalization of QED at 1-loop}

Our first example is the 1-loop renormalization of QED in three different schemes: minimal subtraction (MS), modified minimal subtraction $(\overline{\mathrm{MS}})$ and on-shell (OS). This calculation should be familiar to every QFT practitioner and can be found in many books, e.g. [38] which we will follow here.

We start with

$$
\begin{aligned}
\mathcal{L}_{\mathrm{QED}} & =\mathcal{L}_{\mathrm{R}, \mathrm{QED}}+\mathcal{L}_{\mathrm{CT}} \\
\mathcal{L}_{\mathrm{R}, \mathrm{QED}} & =-\frac{1}{4} F_{\mu \nu} F^{\mu \nu}-\frac{1}{2 \xi}\left(\partial^{\mu} A_{\mu}\right)^{2}+\bar{\psi}(i \not \partial-m) \psi+e \bar{\psi} A \bar{\psi} \\
\mathcal{L}_{\mathrm{CT}} & =-\left(Z_{A}-1\right) \frac{1}{4} F_{\mu \nu} F^{\mu \nu}-\frac{1}{2 \xi}\left(Z_{A} Z_{\xi}^{-1}-1\right)\left(\partial^{\mu} A_{\mu}\right)^{2} \\
& +\left(Z_{\psi}-1\right) \bar{\psi} i \not \partial \psi-\left(Z_{\psi} Z_{m}-1\right) m \bar{\psi} \psi+\left(Z_{\psi} Z_{A}^{1 / 2} Z_{e}-1\right) e \bar{\psi} \gamma^{\mu} \psi A_{\mu} .
\end{aligned}
$$

where $\mathcal{L}_{\mathrm{R}, \mathrm{QED}}$ contains only renormalized quantities and $\mathcal{L}_{\mathrm{CT}}$ provides the counter terms. The renormalization constants are defined as

$$
e_{0}=Z_{e} e, \quad m_{0}=Z_{m} m, \quad \xi_{0}=Z_{\xi} \xi, \quad \psi_{0}=Z_{\psi}^{1 / 2} \psi, \quad A_{0, \mu}=Z_{A}^{1 / 2} A_{\mu},
$$

\footnotetext{
11 When FEynHelpers is loaded, the greeting message contains the sentence "Have a look at the supplied examples". Clicking on the word "examples" opens the directory with sample calculations.

12 https://github.com/FeynCalc/feynhelpers/tree/master/Examples
} 
where the subscript 0 denotes bare quantities. From the Ward identities for the photon propagator and for the electron-photon vertex we obtain that

$$
Z_{\xi}=Z_{A}, \quad Z_{e}=1 / \sqrt{Z_{A}}
$$

Our task is, therefore, to determine $Z_{A}, Z_{\psi}$ and $Z_{m}$ at 1-loop in three different schemes.

In the minimal subtraction schemes we demand that the two-point functions of the electron and the photon are finite at 1-loop, i.e.

$$
\begin{aligned}
& i \Gamma_{R}^{\psi \bar{\psi}}(p,-p)=\longrightarrow+\underset{\longrightarrow}{\longrightarrow}+\longrightarrow \boldsymbol{\longrightarrow}+\mathcal{O}\left(\alpha^{2}\right) \stackrel{!}{=} \text { finite } \\
& \leftrightarrow \Gamma_{R}^{\psi \bar{\psi}}(p,-p)=(\not p-m)+\Sigma(\not p)+\left(Z_{\psi}^{\mathrm{MS} / \overline{\mathrm{MS}}}-1\right) \not p \\
& -\left(Z_{\psi}^{\mathrm{MS} / \overline{\mathrm{MS}}} Z_{m}^{\mathrm{MS} / \overline{\mathrm{MS}}}-1\right) m+\mathcal{O}\left(\alpha^{2}\right) \stackrel{!}{=} \text { finite }
\end{aligned}
$$

and

$$
\begin{aligned}
-i\left(\Gamma_{R}^{A A}\right)^{\mu \nu}(q,-q) & =\stackrel{\mu}{\sim}+\stackrel{\mu}{\sim} \nu^{\nu}+\mu \sim \sim^{\nu}+\mathcal{O}\left(\alpha^{2}\right) \stackrel{!}{=} \text { finite } \\
\leftrightarrow\left(\Gamma_{R}^{A A}\right)^{\mu \nu}(q,-q) & =\left(q^{2} g^{\mu \nu}-q^{\mu} q^{\nu}(1-\xi)\right)+\Pi^{\mu \nu}(q) \\
& +\left(q^{2} g^{\mu \nu}-q^{\mu} q^{\nu}\right)\left(Z_{A}^{\mathrm{MS} / \overline{\mathrm{MS}}}-1\right)+\mathcal{O}\left(\alpha^{2}\right) \stackrel{!}{=} \text { finite }
\end{aligned}
$$

Using that

$$
\begin{aligned}
& \underset{\sim}{\stackrel{\sim}{\sim}} \equiv i \Sigma(\not p)=i\left(\not p \Sigma_{V}\left(p^{2}\right)+m \Sigma_{S}\left(p^{2}\right)\right), \\
& \sim \sim^{\nu} \equiv-i \Pi^{\mu \nu}(q)=-i\left(q^{2} g^{\mu \nu}-q^{\mu} q^{\nu}\right) \Pi\left(q^{2}\right)
\end{aligned}
$$

and $Z_{i}=1+\delta_{i}+\mathcal{O}\left(\alpha^{2}\right)$ we can rewrite Eqs. (15) and (17) as

$$
\begin{array}{r}
\not p\left(\delta_{\psi}^{\mathrm{MS} / \overline{\mathrm{MS}}}+\Sigma_{V}\left(p^{2}\right)\right)-m\left(\delta_{\psi}^{\mathrm{MS} / \overline{\mathrm{MS}}}+\delta_{m}^{\mathrm{MS} / \overline{\mathrm{MS}}}-\Sigma_{S}\left(p^{2}\right)\right)+\mathcal{O}\left(\alpha^{2}\right) \stackrel{!}{=} \text { finite } \\
\Pi\left(q^{2}\right)+\delta_{A}^{\mathrm{MS} / \overline{\mathrm{MS}}}+\mathcal{O}\left(\alpha^{2}\right) \stackrel{!}{=} \text { finite }
\end{array}
$$

where we dropped the manifestly finite terms $(\not p-m)$ and $\left(q^{2} g^{\mu \nu}-q^{\mu} q^{\nu}(1-\xi)\right)$, since in minimal subtraction schemes we are interested only in subtracting the singularity $1 / \epsilon$ (MS scheme) or $1 / \epsilon-\gamma_{E}+\log (4 \pi)(\overline{\mathrm{MS}}$ scheme). 
The renormalization condition for the electron-photon vertex reads

$$
\begin{aligned}
i e\left(\Gamma_{R}^{A \psi \bar{\psi}}\right)^{\mu}\left(p_{1},-p_{2}\right) & =\underbrace{\mu}_{(22)}+3^{\mu}+\mathcal{O}\left(\alpha^{2}\right) \stackrel{!}{=} \text { finite } \\
\leftrightarrow\left(\Gamma_{R}^{A \psi \bar{\psi}}\right)^{\mu}\left(p_{1},-p_{2}\right) & =\gamma^{\mu}+\mathcal{V}^{\mu}\left(p_{1}, p_{2}\right) \\
& +\left(Z_{\psi}^{\mathrm{MS} / \overline{\mathrm{MS}}} \sqrt{Z_{A}^{\mathrm{MS} / \overline{\mathrm{MS}}}} Z_{e}^{\mathrm{MS} / \overline{\mathrm{MS}}}-1\right) \gamma^{\mu}+\mathcal{O}\left(\alpha^{2}\right) \stackrel{!}{=} \text { finite }
\end{aligned}
$$

where

$$
\overbrace{}^{\mu} \equiv i e \mathcal{V}^{\mu}\left(p_{1}, p_{2}\right)
$$

The condition given in Eq. (23) is equivalent to

$$
\mathcal{V}^{\mu}\left(p_{1}, p_{2}\right)+\left(\frac{1}{2} \delta_{A}^{\mathrm{MS} / \overline{\mathrm{MS}}}+\delta_{e}^{\mathrm{MS} / \overline{\mathrm{MS}}}+\delta_{\psi}^{\mathrm{MS} / \overline{\mathrm{MS}}}\right) \gamma^{\mu} \stackrel{!}{=} \text { finite. }
$$

Since $Z_{e}$ has already been fixed by the Ward's identity, it is not necessary to explicitly evaluate the vertex function. On the other hand, nothing prevents us from doing so as a cross-check for the whole calculation.

In the on-shell scheme one demands that the renormalized self-energies satisfy

$$
\begin{array}{r}
\lim _{p^{2} \rightarrow m^{2}}\left(\frac{1}{\not p-m} \Gamma_{R}^{\psi \psi} \bar{\psi}(p,-p) u(p) \stackrel{!}{=} u(p)\right), \\
\lim _{q^{2} \rightarrow 0}\left(\frac{\left(\Gamma_{R}^{A A}\right)^{\mu \nu}(q,-q)}{q^{2}} \varepsilon_{\nu}(q) \stackrel{!}{=}-\varepsilon^{\mu}(q)\right) .
\end{array}
$$

It is easy to show that this corresponds to the following conditions

$$
\begin{aligned}
& \delta_{m}^{\mathrm{OS}} \stackrel{!}{=} \Sigma_{S}\left(m^{2}\right)+\Sigma_{V}\left(m^{2}\right), \\
& \delta_{\psi}^{\mathrm{OS}} \stackrel{!}{=}-\Sigma_{V}\left(m^{2}\right)+2 m^{2}\left(\Sigma_{S}^{\prime}\left(m^{2}\right)+\Sigma_{V}^{\prime}\left(m^{2}\right)\right), \\
& \delta_{A}^{\mathrm{OS}} \stackrel{!}{=}-\lim _{q^{2} \rightarrow 0} \frac{\partial}{\partial q^{2}}\left(q^{2} \Pi\left(q^{2}\right)\right) .
\end{aligned}
$$


The on-shell condition for the vertex is given by

$$
\bar{u}(p)\left(\Gamma_{R}^{A \psi \bar{\psi}}\right)^{\mu}(p,-p) u(p)=\bar{u}(p) \gamma^{\mu} u(p),
$$

with $p^{2}=m^{2}$. It should also be remarked that, apart from the renormalization, the evaluation of the on-shell vertex function allows us to extract some beautiful piece of physics. Owing to Lorentz invariance, the vertex function sandwiched between two spinors with $p_{1} \neq p_{2}$ can be parametrized as

$$
\bar{u}\left(p_{2}\right)\left(\Gamma_{R}^{A \psi \bar{\psi}}\right)^{\mu}\left(p_{1},-p_{2}\right) u\left(p_{1}\right) \stackrel{!}{=} \bar{u}\left(p_{2}\right)\left(\gamma^{\mu} F_{1}\left(q^{2}\right)+\frac{i \sigma^{\mu \nu} q_{\nu}}{2 m} F_{2}\left(q^{2}\right)\right) u\left(p_{1}\right),
$$

with $q \equiv p_{2}-p_{1}$ and $\sigma^{\mu \nu}=\frac{i}{2}\left[\gamma^{\mu}, \gamma^{\nu}\right]$. Here, $F_{2}(0)$ is the 1-loop quantum correction to the anomalous magnetic moment of the electron in QED that was first obtained by Schwinger [39] in 1948

$$
\frac{g-2}{2}=F_{2}(0)=\frac{\alpha}{2 \pi}
$$

and is often considered to be one of the greatest triumphs of QFT.

For $p_{2}=p_{1} \equiv p$ the term proportional to $F_{2}(0)$ vanishes and we are left with $F_{1}(0)$ only, i.e.

$$
\begin{aligned}
& \bar{u}(p)\left(\Gamma_{R}^{A \psi \bar{\psi}}\right)^{\mu}(p,-p) u(p)= \\
& \bar{u}(p) \gamma^{\mu} u(p)+\bar{u}(p) \mathcal{V}^{\mu} u(p)+\left(\frac{1}{2} \delta_{A}^{\mathrm{OS}}+\delta_{e}^{\mathrm{OS}}+\delta_{\psi}^{\mathrm{OS}}\right) \bar{u}(p) \gamma^{\mu} u(p)= \\
& =F_{1}(0) \bar{u}(p) \gamma^{\mu} u(p) \stackrel{!}{=} \bar{u}(p) \gamma^{\mu} u(p) .
\end{aligned}
$$

This leads to the renormalization condition

$$
\bar{u}(p) \mathcal{V}^{\mu}(p, p) u(p)=-\left(\frac{1}{2} \delta_{A}^{\mathrm{OS}}+\delta_{e}^{\mathrm{OS}}+\delta_{\psi}^{\mathrm{OS}}\right) .
$$

Now that we fully understand what we want to compute, it is time to carry out the calculation. When doing things by pen and paper, this is usually the most dull part, which however requires great care. With FEYNCALC and FEynHelpers one, of course, still has to pay attention to what one is doing, but the calculation itself can be done much faster.

Although FEYNARTS already contains the full Standard Model Lagrangian including the counter-terms, for this example we choose to create a model 
file that contains only QED. This can be conveniently done via FEYNRULES [4], a MathematicA package that generates Feynman rules out of the given Lagrangian. The reason for doing so is to show how FEYNRules can be chained with FEynARTs, FEynCALC and FEYnHELPERs to generate new models and study their phenomenology, including the determination of the renormalization coefficients in different schemes. The results can be also used as a cross-check for NLOCT [40], a FEYNRULES extension for fully automatic 1-loop renormalization of arbitrary models.

The FEYNRULES-model file QED.fr is already included in FEYNCALC 9.2 and can be found in FeynCalc/Examples/FeynRules/QED. Evaluating GenerateModelQED.m converts the input file QED.fr into a working FEYNARTS model and saves it to FeynCalc/FeynArts/Models/QED. Then we start a new Mathematica kernel and load FeynCalc, FeynArts and FEYNHELPERS in the usual way

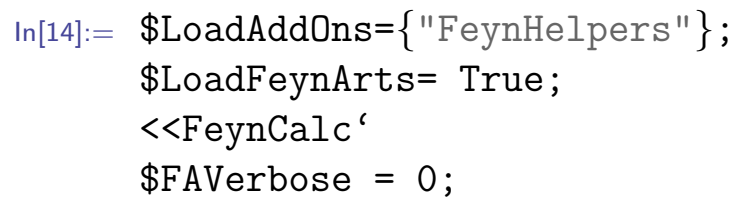

First of all we need to patch the new FEYNARTS QED model in order to make it compatible with FEYNCALC. Since in this calculation we will explicitly distinguish between UV and IR divergences regulated by $\epsilon_{\mathrm{UV}}$ and $\epsilon_{\mathrm{IR}}$, we also need to activate the corresponding option

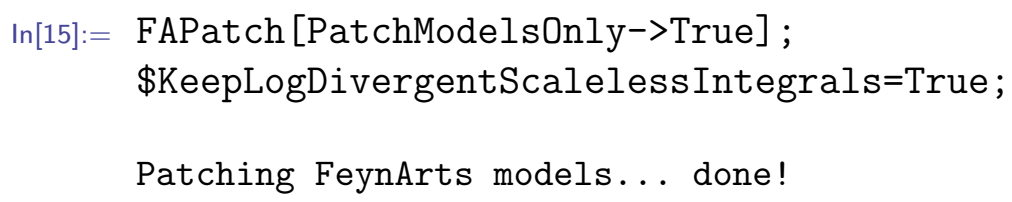

From our QED model we can generate the required 1-loop diagrams and the corresponding counter-terms with FEYNARTS and then evaluate them with FeynCalc and FeynHelpers. The conversion of a FeynArts amplitude into FEYNCALC notation can be conveniently done with FCFAConvert, where we explicitly keep the gauge parameter $\xi$. Our starting point is the electron self-energy $i \Sigma(\not p)$. Tensor reduction with TID allows us to express this amplitude in terms of the Passarino-Veltman functions $A_{0}, B_{0}$ and $C_{0}$, while PaXEvaluateUVIRSplit provides the full analytic result for $i \Sigma(\not p)$. 
According to Eq. 20 we need to determine $\delta_{\psi}$ and $\delta_{m}$ in such a way, that the singularity is subtracted. This can be achieved by adding the amplitudes for the 1-loop self-energy and the counter-term and discarding all the finite terms, which gives

$$
\text { Out[15] }=-i \delta_{\psi} \mathrm{m}_{\mathrm{e}}-i \mathrm{~m}_{\mathrm{e}} \delta_{\mathrm{m}}+\frac{i \alpha \Delta_{\mathrm{UV}}\left(-\mathrm{m}_{\mathrm{e}} \xi_{\mathrm{V}(1)}-3 \mathrm{~m}_{\mathrm{e}}+\xi_{\mathrm{V}(1)} \gamma \cdot \mathrm{p}\right)}{4 \pi}+i \delta_{\psi} \gamma \cdot \mathrm{p}
$$

Equating this to zero and solving for $\delta_{\psi}$ and $\delta_{m}$ we obtain

$$
\begin{aligned}
& \mathrm{Out}[16]=\left\{\delta_{\psi}^{\mathrm{MS}} \rightarrow-\frac{\alpha \xi_{\mathrm{V}(1)}}{4 \pi \varepsilon_{\mathrm{UV}}}, \quad \delta_{\mathrm{m}}^{\mathrm{MS}} \rightarrow-\frac{3 \alpha}{4 \pi \varepsilon_{\mathrm{UV}}}\right\} \\
& \mathrm{Out}[17]=\left\{\delta_{\psi}^{\mathrm{MS}} \rightarrow-\frac{\alpha \Delta_{\mathrm{UV}} \xi_{\mathrm{v}(1)}}{4 \pi}, \delta_{\mathrm{m}}^{---\overline{\mathrm{MS}}} \rightarrow-\frac{3 \alpha \Delta_{\mathrm{UV}}}{4 \pi}\right\}
\end{aligned}
$$

In order to compute these renormalization constants in the on-shell scheme we need to extract $\Sigma_{S}\left(p^{2}\right)$ and $\Sigma_{V}\left(p^{2}\right)$ first. Eq. 28 tells us that the mass renormalization constant follows from the sum of $\Sigma_{S}$ and $\Sigma_{V}$ evaluated at $p^{2}=m^{2}$. To compute this integral we expand $\Sigma_{S}\left(p^{2}\right)+\Sigma_{V}\left(p^{2}\right)$ around $p^{2}=m^{2}$ to zeroth order with PaXSeries and obtain

$$
\text { Out }[18]=\left\{\delta_{\mathrm{m}}^{\mathrm{OS}} \rightarrow-\frac{\alpha\left(3 \log \left(\frac{\mu^{2}}{\mathrm{~m}_{\mathrm{e}}^{2}}\right)+3 \Delta_{\mathrm{UV}}+4\right)}{4 \pi}\right\}
$$

In the same manner we can also compute the values of $\Sigma_{S}^{\prime}\left(m^{2}\right)+\Sigma_{V}^{\prime}\left(m^{2}\right)$ and $\Sigma_{V}\left(m^{2}\right)$. Plugging them into Eq. (29) yields

$$
\text { Out }[19]=\left\{\delta_{\psi}^{\mathrm{OS}} \rightarrow-\frac{\alpha\left(3 \log \left(\frac{\mu^{2}}{\mathrm{~m}_{\mathrm{e}}^{2}}\right)-\Delta_{\mathrm{IR}}\left(\xi_{\mathrm{V}(1)}-3\right)+\Delta_{\mathrm{UV}} \xi_{\mathrm{V}(1)}+4\right)}{4 \pi}\right\}
$$

As is familiar from the literature [41], for $\Delta_{\mathrm{UV}}=\Delta_{\mathrm{IR}}=\Delta$ the parameter $\xi$ drops out, leaving us with a gauge independent fermion wave-function renormalization constant $\delta_{\psi}^{\mathrm{OS}}=\delta_{m}^{\mathrm{OS}}$.

The evaluation of the photon self-energy proceeds similarly. From Eq. (21) we get the renormalization constant $\delta_{A}$ in the minimal subtraction schemes

$$
\text { Out }[20]=\left\{\delta_{\mathrm{A}}^{\mathrm{MS}} \rightarrow-\frac{\alpha}{3 \pi \varepsilon_{\mathrm{UV}}}\right\}
$$




$$
\text { Out }[21]=\left\{\delta_{\mathrm{A}}^{----} \rightarrow-\frac{\alpha \Delta_{\mathrm{UV}}}{3 \pi}\right\}
$$

Then we again use PaXSeries to compute $\lim _{q^{2} \rightarrow 0} \frac{\partial}{\partial q^{2}}\left(q^{2} \Pi\left(q^{2}\right)\right)$ and thus determine $\delta_{A}$ in the on-shell scheme

$$
\text { Out [22] }=\left\{\delta_{\mathrm{A}}^{\mathrm{OS}} \rightarrow-\frac{\alpha\left(\log \left(\frac{\mu^{2}}{\mathrm{~m}_{\mathrm{e}}^{2}}\right)+\Delta_{\mathrm{UV}}\right)}{3 \pi}\right\}
$$

Let us now also do some calculations with the vertex function. Although TID can of course reduce it into basic scalar integrals, the resulting expression will be huge, as we keep the kinematics completely general. We can, however, obtain a much more compact expression by sticking to coefficient functions, i.e. by activating the option UsePaVeBasis. Furthermore, as we are interested only in the UV-part of the whole expression, it is sufficient (and also much faster) to use PaXEvaluateUV instead of PaXEvaluateUVIRSplit. Then the UV-part of the vertex diagram reads

$$
\text { Out[23] }=\frac{i \alpha e \gamma^{\text {Lor1 }} \xi_{\mathrm{V}(1)}}{4 \pi \varepsilon_{\mathrm{UV}}}
$$

and with Eq. (23) we arrive to

$$
\text { Out[24] }=-\frac{2 \pi \varepsilon_{\mathrm{UV}}\left(\delta_{\mathrm{A}}+2\left(\delta_{\psi}+\delta_{\mathrm{e}}\right)\right)+\alpha \xi_{\mathrm{V}(1)}}{4 \pi \varepsilon_{\mathrm{UV}}}=0
$$

Plugging our results for $\delta_{\psi}$ and $\delta_{m}$ into this relation yields

$$
\text { Out[25] }=\delta_{\mathrm{A}}+2 \delta_{\mathrm{e}}=0
$$

which explicitly confirms the relation $Z_{e}=1 / \sqrt{Z_{A}}$ in the minimal subtraction schemes.

To confirm this relation also in the on-shell scheme we need to look at the vertex function sandwiched between two spinors with their 4-momenta being put on-shell. At first, we choose the momenta to be $p_{1}$ and $p_{2}$ with $p_{1} \neq p_{2}$. After having applied Gordon decomposition

$$
\bar{u}\left(p_{2}\right) \gamma^{\mu} u\left(p_{1}\right)=\bar{u}\left(p_{2}\right)\left(\frac{\left(p_{1}+p_{2}\right)^{\mu}}{2 m}+\frac{i \sigma^{\mu \nu}\left(p_{2}-p_{1}\right)_{\nu}}{2 m}\right) u(p)
$$

we can bring the amplitude to the form dictated by Eq. (32). As neither the counter-term nor the tree-level vertex contain a term proportional to $\sigma^{\mu \nu}$, the 1-loop vertex amplitude alone is sufficient to extract $F_{2}\left(q^{2}\right)$ 


$$
\begin{aligned}
\text { Out }[26]= & 16 \pi^{3} \alpha \mathrm{m}_{\mathrm{e}}^{2}\left(2 \mathrm{C}_{1}\left(\mathrm{~m}_{\mathrm{e}}^{2}, 2 \mathrm{~m}_{\mathrm{e}}^{2}-2(\mathrm{p} 1 \cdot \mathrm{p} 2), \mathrm{m}_{\mathrm{e}}^{2}, 0, \mathrm{~m}_{\mathrm{e}}^{2}, \mathrm{~m}_{\mathrm{e}}^{2}\right)+(\mathrm{D}-2)\left(\mathrm{C}_{11}(\right.\right. \\
& \left.\mathrm{m}_{\mathrm{e}}^{2}, 2 \mathrm{~m}_{\mathrm{e}}^{2}-2(\mathrm{p} 1 \cdot \mathrm{p} 2), \mathrm{m}_{\mathrm{e}}^{2}, 0, \mathrm{~m}_{\mathrm{e}}^{2}, \mathrm{~m}_{\mathrm{e}}^{2}\right)+\mathrm{C}_{12}\left(\mathrm{~m}_{\mathrm{e}}^{2}, 2 \mathrm{~m}_{\mathrm{e}}^{2}-2(\mathrm{p} 1 \cdot \mathrm{p} 2)\right. \\
& \left.\left.\left.\mathrm{m}_{\mathrm{e}}^{2}, \quad 0, \mathrm{~m}_{\mathrm{e}}^{2}, \mathrm{~m}_{\mathrm{e}}^{2}\right)\right)\right)
\end{aligned}
$$

Evaluating it at $q^{2}=0$ we recover the famous result for $F_{2}(0)=\frac{g-2}{2}$

$$
\begin{aligned}
\operatorname{In}[27]:= & \text { PaXEvaluateUVIRSplit }[\operatorname{tmp}[15] / .\{\operatorname{FCI}[\operatorname{SPD}[\mathrm{p} 1, \mathrm{p} 2]] \rightarrow \operatorname{SMP}[" \\
& \left.\left.\left.\mathrm{m}_{-} \mathrm{e}\right]^{\wedge} 2\right\}, \text { PaXImplicitPrefactor } \rightarrow 1 /(2 \mathrm{Pi})^{\wedge} \mathrm{D}\right] \\
\text { Out }[27]= & \frac{\alpha}{2 \pi}
\end{aligned}
$$

When we set $p_{1}=p_{2} \equiv p$, the term proportional to $F_{2}(0)$ vanishes. Extracting $F_{1}(0)$ from $\bar{u}(p)\left(\Gamma_{R}^{A \psi \psi} \bar{\psi}\right)^{\mu}(p,-p) u(p)$ according to Eq. (34) tells us that

$$
\text { Out[28] }=-\frac{2 \pi \varepsilon_{\mathrm{UV}}\left(\delta_{\mathrm{A}}+2\left(\delta_{\psi}+\delta_{\mathrm{e}}\right)\right)+\alpha \xi_{\mathrm{V}(1)}}{4 \pi \varepsilon_{\mathrm{UV}}}=0
$$

and hence again

$$
\text { Out[29] }=\delta_{\mathrm{A}}+2 \delta_{\mathrm{e}}=0
$$

as it should.

This concludes the 1-loop renormalization of QED in 3 different schemes, carried out with FeynCalc, FeynHelpers, FeynArts and FeynRules. Needless to say that similar calculations can also be done for more complicated theories (e.g., new models for physics beyond the Standard Model).

\subsection{Quark-gluon vertex expanded in the relative momentum squared at 1-loop}

The ability of FEYNHELPERS to distinguish between dimensionally regulated UV and IR divergences at 1-loop and to expand coefficient functions in their parameters can be also used to reproduce parts of the 1-loop matching between QCD and nonrelativistic QCD (NRQCD) 42. NRQCD is an EFT that is widely used to describe production and decay of heavy quarkonia. In [2] we have already demonstrated how FEYNCALC can be employed for the tree-level matching between both theories. Here we concentrate on the 1-loop matching by following the famous work of Manohar [37], where on the QCD side of the matching the on-shell vertex function was evaluated using 
background field formalism [43, 44] and expanded up to the first order in the relative momentum squared.

As FEYNARTs does not contain a model for background-field QCD, we chose to implement it ourselves using FEYNRULES and to include the model file QCDBGF.fr to FEYNCALC 9.2 2 , A working FEYNARTS model can be generated via GenerateModelQCDBGF.m.

The abelian and nonabelian diagrams can be parametrized as
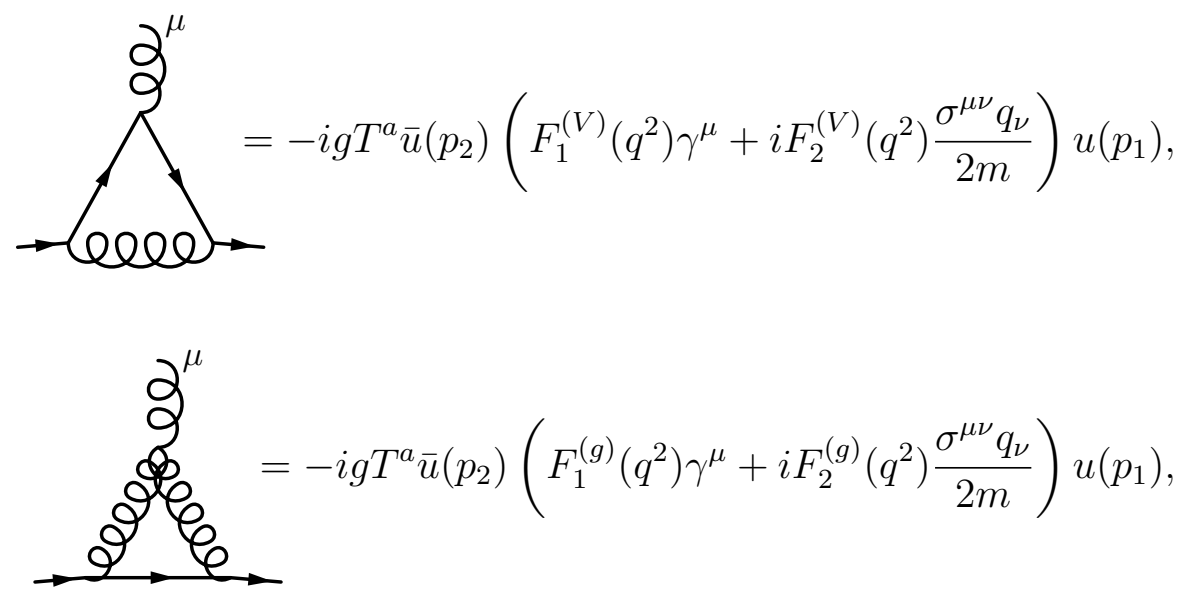

where $q \equiv p_{2}=p_{1}$. Our goal is to compute the form-factors $F_{1 / 2}^{(V)}\left(q^{2}\right)$ and $F_{1 / 2}^{(g)}\left(q^{2}\right)$ expanded up to $\mathcal{O}\left(q^{2} / m^{2}\right)$. In this case the whole calculation can be essentially split into 5 distinct steps

1. Generate the diagrams with FEYNARTs and prepare amplitudes for FEYNCALC.

2. Perform tensor decomposition of 1-loop integrals and simplify the Dirac algebra.

3. Apply Gordon decomposition.

4. Expand the amplitudes in $q^{2}$ with FeynHeLPers.

5. Extract the form-factors.

Someone familiar with FEYNCALC can prepare the corresponding code with minimal effort. Applying it to each of the diagrams we find that $F_{1}^{(V)}\left(q^{2}\right)$ and $F_{2}^{(V)}\left(q^{2}\right)$ are given by

${ }^{13}$ The model file is located in FeynCalc/Examples/FeynRules/QCDBGF. 


$$
\begin{aligned}
\text { Out }[30]= & \frac{1}{\pi} \alpha_{\mathrm{s}}\left(\mathrm{C}_{\mathrm{F}}-\frac{\mathrm{C}_{\mathrm{A}}}{2}\right)\left(\frac{\Delta_{\mathrm{IR}}}{2}-\frac{\mathrm{q} 2 \Delta_{\mathrm{IR}}}{6 \mathrm{~m}^{2}}+\frac{3}{4} \log \left(\frac{\mu^{2}}{\mathrm{~m}^{2}}\right)-\frac{\mathrm{q} 2\left(4 \log \left(\frac{\mu^{2}}{\mathrm{~m}^{2}}\right)+3\right)}{24 \mathrm{~m}^{2}}+\right. \\
& \left.\frac{\Delta_{\mathrm{UV}}}{4}+1\right)
\end{aligned}
$$

and

$$
\text { Out[31] }=\frac{\left(\frac{\mathrm{q} 2}{12 \mathrm{~m}^{2}}+\frac{1}{2}\right) \alpha_{\mathrm{s}}\left(\mathrm{C}_{\mathrm{F}}-\frac{\mathrm{C}_{\mathrm{A}}}{2}\right)}{\pi}
$$

respectively, while the nonabelian form-factors $F_{1}^{(g)}\left(q^{2}\right)$ and $F_{2}^{(g)}\left(q^{2}\right)$ read

$$
\text { Out[32] }=\frac{1}{8 \pi} \mathrm{C}_{\mathrm{A}} \alpha_{\mathrm{s}}\left(2 \Delta_{\mathrm{IR}}-\frac{3 \mathrm{q} 2 \Delta_{\mathrm{IR}}}{2 \mathrm{~m}^{2}}+3 \log \left(\frac{\mu^{2}}{\mathrm{~m}^{2}}\right)-\frac{\mathrm{q} 2\left(3 \log \left(\frac{\mu^{2}}{\mathrm{~m}^{2}}\right)+2\right)}{2 \mathrm{~m}^{2}}+\Delta_{\mathrm{UV}}+4\right)
$$

and

$$
\text { Out [33] }=\frac{1}{8 \pi} \mathrm{C}_{\mathrm{A}} \alpha_{\mathrm{s}}\left(2 \Delta_{\mathrm{IR}}+\frac{2 \mathrm{q} 2 \Delta_{\mathrm{IR}}}{\mathrm{m}^{2}}+2\left(\log \left(\frac{\mu^{2}}{\mathrm{~m}^{2}}\right)+3\right)+\frac{\mathrm{q} 2\left(2 \log \left(\frac{\mu^{2}}{\mathrm{~m}^{2}}\right)+1\right)}{\mathrm{m}^{2}}\right)
$$

where q2 $\equiv q^{2}$. To compare this to the results presented in [37, we need to switch from $D=4-2 \epsilon$ to $D=4-\epsilon$ via $1 / \epsilon \rightarrow 2 / \epsilon$ and eliminate $\gamma_{E}$ and $\log (4 \pi)$ by substituting $\mu^{2}$ with $\mu^{2} \frac{e^{\gamma} E}{4 \pi}$. After doing so we precisely recover Eqs. (24)-(25) and Eqs. (29)-(30) from [37.

\subsection{Higgs decay to two gluons}

Let us consider the partial decay width of the Higgs boson into two gluons via a top-quark loop ${ }^{14}$. With FeynCalc, FeynARTs and FeynHelpers the calculation is very similar to the two previous examples, the main difference being that for the generation of the diagrams we use SM.mod, the default Standard Model implementation shipped with FEYnARTs. As the amplitude for this process is finite, it is sufficient to use PaXEvaluate only. Squaring the amplitude, averaging over the polarizations of the on-shell gluons and multiplying by the phase space factor we arrive at the following expression for $\Gamma(H \rightarrow g g)$

\footnotetext{
${ }^{14}$ The calculation of this process with PACKAGE-X alone can be found in the official tutorial of the package, Sec 5.2.
} 

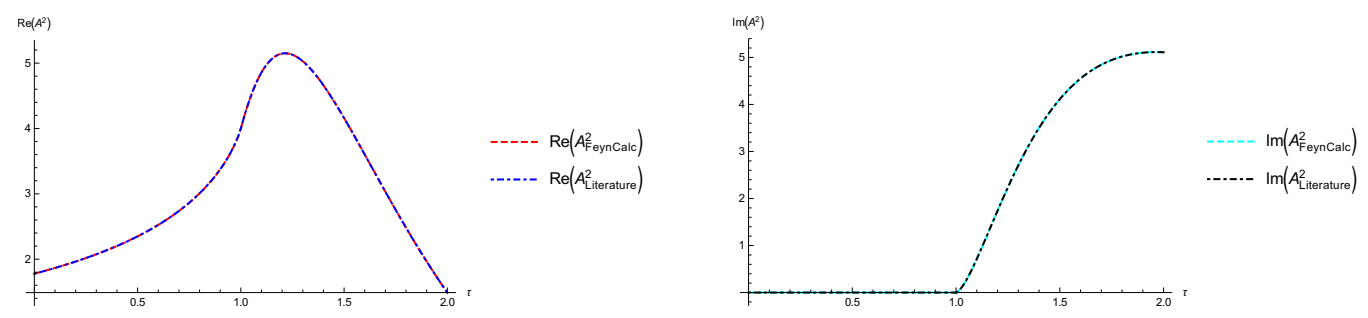

Figure 1: Real and imaginary parts of $A(\tau)$ from the literature and from the result obtained with FeYnHELPERS.

$$
\text { Out[34] }=\frac{\mathrm{G}_{\mathrm{F}} \mathrm{m}_{\mathrm{H}}^{3} \alpha_{\mathrm{s}}^{2}\left((\tau-1) \log ^{2}\left(2\left(\sqrt{\frac{\tau-1}{\tau}}-1\right) \tau+1\right)-4 \tau\right)^{2}}{256 \sqrt{2} \pi^{3} \tau^{4}}
$$

with $\tau=\frac{m_{H}^{2}}{4 m_{t}^{2}}$, where $m_{H}$ denotes the Higgs mass, $m_{t}$ the mass of the top quark and $G_{F}$ stands for the Fermi constant. According to the literature [45], $\Gamma(H \rightarrow g g)$ (taking into account all quark flavors) can be written as

$$
\Gamma(H \rightarrow g g)=\frac{G_{F} \alpha_{s}^{2}}{36 \sqrt{2} \pi^{3}} m_{H}^{3}\left|\frac{3}{4} \sum_{\substack{Q=u, d, c, s, t, b}} A_{Q}\left(\tau_{Q}\right)\right|^{2},
$$

where $\tau_{Q}=\frac{m_{H}^{2}}{4 m_{Q}^{2}}$ and $A_{Q}$ is defined as

$$
A_{Q}\left(\tau_{Q}\right)=\frac{2}{\tau_{Q}^{2}}\left[\tau_{Q}+\left(\tau_{Q}-1\right) f\left(\tau_{Q}\right)\right]
$$

with

$$
f\left(\tau_{Q}\right)= \begin{cases}\arcsin ^{2}\left(\sqrt{\tau_{Q}}\right) & \text { if } \tau_{Q} \leq 1 \\ -\frac{1}{4}\left[\log \left(\frac{1+\sqrt{1+\tau^{-1}}}{1-\sqrt{1-\tau^{-1}}}\right)-i \pi\right]^{2} & \text { if } \tau_{Q}>1\end{cases}
$$

Depending on the quark mass, one can have $\tau_{Q} \leq 1$ or $\tau_{Q}>1$, while for the top quark and the known Higgs mass only the former case is relevant. Nevertheless, extracting $A_{t}^{2}\left(\tau_{t}\right) \equiv A^{2}(\tau)$ from our result and comparing it to Eq. (40) we can convince ourselves (c.f. Fig. 1) that the analytic expression returned by PACKAGE-X is indeed valid both for $\tau \leq 1$ and $\tau>1$, such that our result is correct. 


\subsection{2-loop self-energies in massless $Q E D$}

Our last example deals with the photon and electron self-energies (with full gauge dependence) in massless QED at 2-loops. This requires evaluation of six 2-loop diagrams

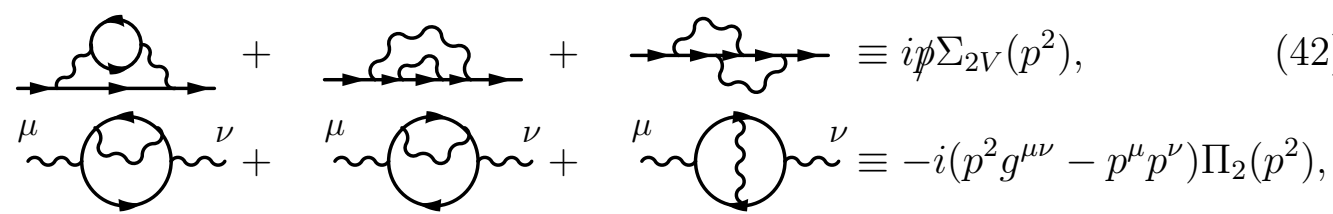

that can be rewritten in terms of only two master integrals. Here we need to handle the amplitudes in a slightly different way, as compared to the previous examples. In particular, for the decomposition of 2-loop tensor integrals we use FCMultiLoopTID instead of TID, that works only at 1-loop. IBP-reduction with FIREBurn is then used to reduce all the resulting scalar integrals into two master integrals. These main steps can be summarized

in only three lines of code for each class of diagrams. For definiteness, let us consider self-energy amplitudes from Eq. (42) and denote the expression obtained from FEYNARTS and processed with FCFAConvert with ampsSE. The sum of the amplitudes depends on the loop momenta $l_{1}$ and $l_{2}$ and the external momentum $p$. With

$$
\begin{aligned}
\ln [35]:= & \text { ampsSE1 = (ampsSE /. DiracTrace } \rightarrow \text { Tr }) / / \\
& \text { FCMultiLoopTID }[\#,\{11,12\}] \& / / \text { DiracSimplify }
\end{aligned}
$$

we evaluate Dirac traces, carry out the tensor decomposition and simplify the resulting Dirac structures. Then, in

$$
\ln [36]:=\operatorname{ampsSE} 2=\operatorname{ampsSE} 1 / / \operatorname{FDS}[\#, 11,12] \&
$$

we use FDS to simplify loop integrals by shifting their loop momenta. The IBP-reduction is started by applying FIREBurn to the resulting expression

$$
\begin{aligned}
\ln [37]:= & \operatorname{ampsSE} 3=\text { FIREBurn [ampsSE2 },\{11,12\},\{p\}] / / \text { FDS }[\#, \\
& 11,12] \&
\end{aligned}
$$

Finally, after sorting terms using FEYNCALC's Collect2 (more advanced version of MATHEMATICA's Collect) 


$$
\begin{aligned}
\ln [38]:= & \text { ampsSE4 }=\text { ampsSE3//Collect2 }[\#,\{\text { FeynAmpDenominator }\}, \\
& \text { Factoring } \rightarrow \text { FullSimplify] \& }
\end{aligned}
$$

and factoring out $i \not p$

$$
\ln [39]:=\text { resSE=Cancel }[\operatorname{ampsSE} 4 /(-\mathrm{I} \text { FCI }[(\mathrm{GSD}[\mathrm{p}])])] / / \text { FullSimplify }
$$

we find that the 2-loop contribution ${ }^{15}$ to $\Sigma_{2 V}\left(p^{2}\right)$ equals

$$
\text { Out }[39]=\frac{(D-2) \mathrm{e}^{4}\left(\frac{2\left((\mathrm{D}-6)(\mathrm{D}-3)(3 \mathrm{D}-8) \xi_{\mathrm{A}}^{2}-\mathrm{D}((\mathrm{D}-9) \mathrm{D}+6)-40\right)}{11^{2} \cdot(11-12)^{2} \cdot(12-\mathrm{p})^{2}}-\frac{(\mathrm{D}-6)(\mathrm{D}-4) \mathrm{p}^{2}\left((\mathrm{D}-2) \xi_{\mathrm{A}}^{2}+\mathrm{D}-6\right)}{11^{2} \cdot 12^{2} \cdot(11-\mathrm{p})^{2} \cdot(12-\mathrm{p})^{2}}\right)}{4(\mathrm{D}-6)(\mathrm{D}-4) \mathrm{p}^{2}}
$$

The treatment of vacuum polarization diagrams proceeds in the same fashion and yields

$$
\text { Out }[40]=\frac{2(\mathrm{D}-2) \mathrm{e}^{4}\left(\frac{4(\mathrm{D}-3)((\mathrm{D}-4) \mathrm{D}+8)}{11^{2} \cdot(11-12)^{2} \cdot(12-\mathrm{p})^{2}}-\frac{(\mathrm{D}-4)((\mathrm{D}-7) \mathrm{D}+16) \mathrm{p}^{2}}{11^{2} \cdot 12^{2} \cdot(11-\mathrm{p})^{2} \cdot(12-\mathrm{p})^{2}}\right)}{(\mathrm{D}-4)^{2}(\mathrm{D}-1) \mathrm{p}^{2}}
$$

for $\Pi_{2}\left(p^{2}\right)$.

As expected, the vacuum polarization amplitude is gauge invariant, while the electron self-energy depends on the gauge parameter $\xi$. These results precisely agree with the literature, e.g. Eq. 5.18 and Eq. 5.51 from [46].

\section{Summary}

We have presented the first stable public version of an easy-to-use interface called FEYNHELPERS that seamlessly integrates the 1-loop library of PACKAGE-X and the IBP-reduction mechanism of FIRE into FEYNCALC. With this add-on many types of calculations that were difficult or hardly feasible with FEYNCALC previously, can now be done in a much simpler way. This was demonstrated with four different examples from QED, QCD and Higgs physics. The interface code is open-source and can be modified to accommodate specific requirements. The goals for the future development of FEYNHELPERS are to further improve the integration with PACKAGE-X and FIRE but also to add new interfaces to interesting and useful HEP tools, like LiteRed 29] or FormTracer [4].

\footnotetext{
${ }^{15}$ with $1 /(2 \pi)^{2 D}$ omitted.
} 


\section{Acknowledgments}

The author would like to thank Hiren Patel, the developer of PACKAGE$\mathrm{X}$, and Alexander Smirnov, the developer of FIRE, for many useful explanations on the usage of their respective packages. He appreciates useful feedback provided by Simone Biondini while using an early version of FEYNHELPERS to cross-check analytic results for some loop integrals computed in [48, 49]. Special gratitude also goes to Norbert Kaiser for his help in checking some results obtained with FeynHelpers and to Rolf Mertig and Frederik Orellana for careful reading of this manuscript. Nora Brambilla, Antonio Vairo and Andrey Grozin are acknowledged for interesting discussions and insightful comments.

This work has been supported by the DFG and the NSFC through funds provided to the Sino-German CRC 110 "Symmetries and the Emergence of Structure in QCD" (NSFC Grant No. 11261130311), and by the DFG cluster

of excellence "Origin and structure of the universe" (www . universe-cluster. de).

\section{References}

[1] R. Mertig, M. Böhm, and A. Denner, Feyn Calc - Computer-algebraic calculation of Feynman amplitudes, Comput. Phys. Commun., 64, 345359, (1991).

[2] V. Shtabovenko, R. Mertig, and F. Orellana, New Developments in FeynCalc 9.0, Comput. Phys. Commun., 207, 432-444, (2016), arXiv:1601.01167.

[3] T. Hahn and M. Perez-Victoria, Automatized One-Loop Calculations in 4 and D dimensions, Comput. Phys. Commun., 118, 153-165, (1999), arXiv:hep-ph/9807565.

[4] N. D. Christensen and C. Duhr, FeynRules - Feynman rules made easy, Comput. Phys. Commun., 180, 1614-1641, (2008), arXiv:0806.4194.

[5] V. Shtabovenko, FeynCalc 9, (2016), arXiv:1604.06709.

[6] H. H. Patel, Package-X: A Mathematica package for the analytic calculation of one-loop integrals, Comput. Phys. Commun., 197, 276-290, (2015), arXiv: 1503.01469 
[7] A. V. Smirnov and V. A. Smirnov, FIRE4, LiteRed and accompanying tools to solve integration by parts relations, Comput. Phys. Commun., 184, 2820-2827, (2013), arXiv:1302.5885.

[8] G. Cullen, H. van Deurzen, N. Greiner, G. Heinrich, G. Luisoni, P. Mastrolia, E. Mirabella, G. Ossola, T. Peraro, J. Schlenk, J. F. von SodenFraunhofen, and F. Tramontano, GoSam-2.0: a tool for automated oneloop calculations within the Standard Model and beyond, Eur. Phys. J. C, 74, 8, 3001, (2014), arXiv:1404.7096.

[9] J.-X. Wang, Progress in FDC project, Nucl. Instrum. Meth. A, 534, 241-245, (2004), arXiv:hep-ph/0407058.

[10] G. Belanger, F. Boudjema, J. Fujimoto, T. Ishikawa, T. Kaneko, K. Kato, and Y. Shimizu, GRACE at ONE-LOOP: Automatic calculation of 1-loop diagrams in the electroweak theory with gauge parameter independence checks, Phys. Rept., 430, 117-209, (2006), arXiv:hep-ph/0308080.

[11] M. Tentyukov and J. Fleischer, A Feynman Diagram Analyser DIANA, Comput. Phys. Commun., 132, 124-141, (2000), arXiv:hep-ph/9904258.

[12] G. Passarino and M. Veltman, One Loop Corrections for $e^{+} e^{-}$Annihilation Into $\mu^{+} \mu^{-}$in the Weinberg Model, Nucl. Phys., B160, 151, (1979).

[13] G. Ossola, C. G. Papadopoulos, and R. Pittau, Reducing full one-loop amplitudes to scalar integrals at the integrand level, Nucl. Phys. B, 763, 147-169, (2007), arXiv:hep-ph/0609007.

[14] G. van Oldenborgh, FF - a package to evaluate one-loop Feynman diagrams, Comput. Phys. Commun., 66, 1-15, (1991).

[15] S. Carrazza, R. K. Ellis, and G. Zanderighi, QCDLoop: a comprehensive framework for one-loop scalar integrals, (2016), arXiv:1605.03181.

[16] A. van Hameren, OneLOop: for the evaluation of one-loop scalar functions, Comput. Phys. Commun., 182, 2427-2438, (2011), arXiv:1007.4716. 
[17] G. Cullen, J. P. Guillet, G. Heinrich, T. Kleinschmidt, E. Pilon, T. Reiter, and M. Rodgers, Golem95C: A library for one-loop integrals with complex masses, (2011), arXiv:1101.5595.

[18] J. Fleischer and T. Riemann, A complete algebraic reduction of oneloop tensor Feynman integrals, Phys. Rev. D, 83, 073004, (2011), arXiv:1009.4436.

[19] A. Denner, S. Dittmaier, and L. Hofer, Collier: a fortran-based Complex One-Loop LIbrary in Extended Regularizations, (2016), arXiv: 1604.06792.

[20] A. Denner and S. Dittmaier, Reduction schemes for one-loop tensor integrals, Nucl. Phys. B, 734, 62-115, (2006), arXiv:hep-ph/0509141.

[21] R. K. Ellis and G. Zanderighi, Scalar one-loop integrals for QCD, JHEP, 0802, 002, (2008), arXiv:0712.1851.

[22] P. W. Angel, Y. Cai, N. L. Rodd, M. A. Schmidt, and R. R. Volkas, Testable two-loop radiative neutrino mass model based on an $L L Q d^{c} Q d^{c}$ effective operator, JHEP, 10, 118, (2013), arXiv:1308.0463.

[23] A. Ilakovac and L. Popov, LOOL: Mathematica package for evaluating leading order one loop functions, (2014), arXiv:1407.2727.

[24] K. Chetyrkin and F. Tkachov, Integration by parts: The algorithm to calculate $\beta$-functions in 4 loops, Nucl. Phys. B, 192, 159-204, (1981).

[25] S. Laporta, High-precision calculation of multi-loop Feynman integrals by difference equations, Int. J. Mod. Phys. A, 15, 5087-5159, (2000), arXiv: hep-ph/0102033.

[26] P. A. Baikov, Explicit solutions of the 3-loop vacuum integral recurrence relations, Phys. Lett. B, 385, 404-410, (1996), arXiv: hep-ph/9603267.

[27] A. V. Smirnov and V. A. Smirnov, S-bases as a tool to solve reduction problems for Feynman integrals, Nucl. Phys. Proc. Suppl., 160, 80-84, (2006), arXiv: hep-ph/0606247.

[28] C. Anastasiou and A. Lazopoulos, Automatic Integral Reduction for Higher Order Perturbative Calculations, JHEP, 0407, 046, (2004), arXiv:hep-ph/0404258. 
[29] R. N. Lee, Presenting LiteRed: a tool for the Loop InTEgrals REDuction, (2012), arXiv:1212.2685.

[30] C. Studerus, Reduze - Feynman Integral Reduction in C++, Comput. Phys. Commun., 181, 1293-1300, (2009), arXiv:0912.2546.

[31] A. V. Smirnov, FIRE5: a C++ implementation of Feynman Integral REduction, (2014), arXiv:1408.2372.

[32] J. A. M. Vermaseren, New features of FORM, (2007), arXiv:math-ph/0010025.

[33] R. Mertig and R. Scharf, TARCER - A Mathematica program for the reduction of two-loop propagator integrals, Comput. Phys. Commun., 111, 265-273, (1998), arXiv: hep-ph/9801383.

[34] F. Feng, \$Apart: A Generalized Mathematica Apart Function, Comput. Phys. Commun., 183, 2158-2164, (2012), arXiv:1204.2314.

[35] F. Feng, APart 2: A generalized Mathematica Apart function, Comput. Phys. Commun., 198, 260-261, (2016).

[36] M. R. Fiorentin, FaRe: a Mathematica package for tensor reduction of Feynman integrals, Int. J. Mod. Phys., C27, 1650027, (2015), arXiv:1507.03527.

[37] A. Manohar, The HQET/NRQCD Lagrangian to order $\alpha / m^{3}$, Phys. Rev. D, 56, 230-237, (1997), arXiv:hep-ph/9701294.

[38] M. Bohm, A. Denner, and H. Joos, Gauge theories of the strong and electroweak interaction, Stuttgart, Germany: Teubner, 784 p, (2001).

[39] J. Schwinger, On Quantum-Electrodynamics and the Magnetic Moment of the Electron, Phys. Rev., 73, 416-417, (1948).

[40] C. Degrande, Automatic evaluation of UV and R2 terms for beyond the Standard Model Lagrangians: a proof-of-principle, Comput. Phys. Commun., 197, 239-262, (2015), arXiv:1406.3030.

[41] D. J. Broadhurst, N. Gray, and K. Schilcher, Gauge-invariant on-shellZ 2 in QED, QCD and the effective field theory of a static quark, Zeitschrift für Physik C Particles and Fields, 52, 111-122, (1991). 
[42] G. T. Bodwin, E. Braaten, and G. P. Lepage, Rigorous QCD Analysis of Inclusive Annihilation and Production of Heavy Quarkonium, Phys. Rev. D, 51, 1125-1171, (1995), arXiv:hep-ph/9407339.

[43] L. Abbott, The background field method beyond one loop, Nucl. Phys. B, 185, 189-203, (1981).

[44] L. Abbott, Introduction to the Background Field Method, Acta Phys. Polon., B13, 33, (1982).

[45] M. Spira, A. Djouadi, D. Graudenz, and P. M. Zerwas, Higgs boson production at the LHC, Nucl. Phys. B, 453, 17-82, (1995), arXiv:hep-ph/9504378.

[46] A. Grozin, Lectures on QED and QCD, (2007), arXiv:hep-ph/0508242.

[47] A. K. Cyrol, M. Mitter, and N. Strodthoff, FormTracer - A Mathematica Tracing Package Using FORM, (2016), arXiv:1610.09331.

[48] S. Biondini, N. Brambilla, M. A. Escobedo, and A. Vairo, CP asymmetry in heavy Majorana neutrino decays at finite temperature: the nearly degenerate case, JHEP, 03, 191, (2016), arXiv:1511.02803.

[49] S. Biondini, N. Brambilla, and A. Vairo, CP asymmetry in heavy Majorana neutrino decays at finite temperature: the hierarchical case, JHEP, 09, 126, (2016), arXiv:1608.01979. 